\title{
Axonal Projection Patterns of the Dorsal Interneuron Populations in the Embryonic Hindbrain
}

\author{
Dana Hirsch ${ }^{1,2}$, Ayelet Kohl'1, Yuan Wang ${ }^{3}$ and Dalit Sela-Donenfeld ${ }^{1 *}$ \\ ${ }^{1}$ Koret School of Veterinary Medicine, The Robert H. Smith Faculty of Agriculture, Food and Environment, The Hebrew \\ University of Jerusalem, Rehovot, Israel, ${ }^{2}$ Department of Veterinary Resources, Weizmann Institute of Science, Rehovot, \\ Israel, ${ }^{3}$ Department of Biomedical Sciences, Program in Neuroscience, College of Medicine, Florida State University, \\ Tallahassee, FL, United States
}

OPEN ACCESS

Edited by:

Matías Hidalgo-Sánchez, University of Extremadura, Spain

Reviewed by: Luis Puelles,

University of Murcia, Spain Bernd Fritzsch,

The University of lowa, United States

*Correspondence:

Dalit Sela-Donenfeld dalit.seladon@mail.huji.ac.il

Received: 11 October 2021 Accepted: 29 November 2021 Published: 24 December 2021

Citation:

Hirsch D, Kohl A, Wang Y and Sela-Donenfeld D (2021) Axonal Projection Patterns of the Dorsal Interneuron Populations

in the Embryonic Hindbrain.

Front. Neuroanat. 15:793161. doi: 10.3389/fnana.2021.793161
Unraveling the inner workings of neural circuits entails understanding the cellular origin and axonal pathfinding of various neuronal groups during development. In the embryonic hindbrain, different subtypes of dorsal interneurons (dINs) evolve along the dorsal-ventral (DV) axis of rhombomeres and are imperative for the assembly of central brainstem circuits. dINs are divided into two classes, class A and class B, each containing four neuronal subgroups ( $\mathrm{dA} 1-4$ and $\mathrm{dB} 1-4)$ that are born in well-defined DV positions. While all interneurons belonging to class A express the transcription factor Olig3 and become excitatory, all class B interneurons express the transcription factor Lbx1 but are diverse in their excitatory or inhibitory fate. Moreover, within every class, each interneuron subtype displays its own specification genes and axonal projection patterns which are required to govern the stage-by-stage assembly of their connectivity toward their target sites. Remarkably, despite the similar genetic landmark of each dINs subgroup along the anterior-posterior (AP) axis of the hindbrain, genetic fate maps of some $\mathrm{dA} / \mathrm{dB}$ neuronal subtypes uncovered their contribution to different nuclei centers in relation to their rhombomeric origin. Thus, DV and AP positional information has to be orchestrated in each $\mathrm{dA} / \mathrm{dB}$ subpopulation to form distinct neuronal circuits in the hindbrain. Over the span of several decades, different axonal routes have been well-documented to dynamically emerge and grow throughout the hindbrain DV and AP positions. Yet, the genetic link between these distinct axonal bundles and their neuronal origin is not fully clear. In this study, we reviewed the available data regarding the association between the specification of early-born dorsal interneuron subpopulations in the hindbrain and their axonal circuitry development and fate, as well as the present existing knowledge on molecular effectors underlying the process of axonal growth.

Keywords: hindbrain, rhombomere, dorsal interneurons, rhombic lip, axonal growth

\section{INTRODUCTION}

The vertebrate central nervous system (CNS) is composed of a vast array of neuronal circuits that are assembled in a stepwise manner to give rise to the enormous diversity of cells and functions. Cell fate acquisition, neural cell migration, and axonal projections are all initiated in the developing neural tube and give rise to the neuronal networks of genetically defined neurons that are 
interconnected within the CNS, as well as with afferent/efferent connections with peripheral targets. It is only with the advent of molecular techniques of lineage tracing, designated gene mutations, and enhanced developmental analyses that have allowed the emerging understanding of the details of neural connectivity assembly. Although many studies uncovered genes that regulate different aspects of the multi-event process that spans from neural specification to circuit formation, there is still missing knowledge regarding how these complex mechanisms are orchestrated to give rise to functional networks, and what goes wrong in neurodevelopmental disorders.

The early development of the CNS starts with a series of swellings followed by elementary division into the forebrain, midbrain, and hindbrain. The hindbrain, which is a highly conserved region across vertebrates, has been traditionally subdivided into the pons, medulla oblongata, and cerebellum, which together compose the brainstem. Notably, a more accurate subdivision of the hindbrain into the prepontine, pontine, retropontine, and medullary sub-domains has been recommended based on cell fate mapping and gene expression analyses (Watson et al., 2019). Positioned between the spinal cord and upper brain, the hindbrain serves as a key relayhub linking the lower and upper parts of the CNS as well as the cranial peripheral nervous system (PNS) via multiple circuits that regulate vital functions such as breathing, fine-tuning movement, blood pressure adjustment, auditory and vestibular sensations and facial movement (Joyner and Zervas, 2006; Stiles and Jernigan, 2010; Champagnat et al., 2011; Nothwang et al., 2015; Hernandez-Miranda et al., 2017; Glover, 2020).

A landmark in hindbrain ontogeny is its segmentation into 7/8 overt units, termed rhombomeres (identified as r1-r8, from anterior to posterior), along the anterior-posterior (AP) extent of the hindbrain anlage (Figure 1A) (Lumsden and Krumlauf, 1996; Moens and Prince, 2002). A more updated classification

Abbreviations: AMB, ambiguous; AP, area postrema; AP, anterioposterior; Atoh1, atonal homolog 1; Ascl1, achaete-scute family bHLH transcription factor 1; AVCN, anteroventral cochlear nucleus; CAM, cell adhesion molecules; cC-VCcaudal vestibular neurons; Cdh, cadherins; $\mathrm{CF}$, climbing fiber; $\mathrm{CN}$, cochlear nuclei; CNS, central nervous system; cVRG, caudal ventral respiratory group; DCC, receptor Deleted in Colorectal Cancer; DCN, dorsal cochlear nuclei; g, dorsal funiculi; dINs, dorsal interneurons; DMRT3, double sex/male abnormal 3; DV, dorsoventral; DVN, descending vestibular nuclei; EW, Edinger-Westphal; FMRP, fragile $\mathrm{X}$ mental retardation protein; FoxB, forkhead box protein B; FP, floor plate; HD, homeodomain; INs, interneurons; ION, inferior olivary nucleus; ITR, intertrigeminal region; KF, Kölliker-Fuse; Lbx1, ladybird homeobox transcription factor; LF, lateral funiculi; LMX1a/b, LIM homeobox transcription factor 1 alpha/beta; LVN, lateral vestibular nuclei; LVST, lateral vestibulospinal tract; MF, mossy fiber; MLD, nucleus mesencephalicus lateralis pars dorsalis; MLF, medial longitudinal fascicle; MNTB, medial nucleus of trapezoid body; MPV, ventral posterior medial; $\mathrm{MSO}$, medial superior olivary; MVN, medial vestibular nuclei; NA, nucleus angularis; Necl, nectin-like proteins; Neurog, neurogenin; NL, nucleus laminaris; NM, nucleus magnocellularis; Npn, neuropilin; NTS, nucleus tractus solitarius; PBN, parabrachial nucleus; PCN, precerebellar nuclei; pFRG, parafacial respiratory group; PGN, pontine gray nucleus; Phox $2 b$, pairedlike homeobox 2b; pLC, pre-locus coeruleus; PNS, peripheral nervous system; Pou4f1, POU domain class 4 transcription factor 1; Pou4f1, POU domain class 4 transcription factor 1; $\operatorname{Pr} 5$, principal trigeminal nucleus; preBötC, preBötzinger Complex; Ptfla, pancreas-specific transcription factor 1a; $r$, rhombomere; RL, rhombic lip-; Robo, roundabout; RTN, retrotrapezoid nucleus; Sema, semaphorin; SOC, superior olivary complex; SON, superior olivary nuclei; Sp5, spinal trigeminal nuclei; Tlx, T cell leukemia homeobox; VCN, ventral cochlear nuclei; Wt1-Wilms tumor protein 1; Zic, zinc-finger of the cerebellum. lists 12 rhombomeres (r0-r11), some of which (r1-r7) are anatomically delimited by constrictive transverse boundaries, while others ( $\mathrm{r} 0, \mathrm{r} 8-\mathrm{r} 11)$, are delimited by molecular and cell lineage parameters and are known as crypto-rhombomeres (also called pseudo-rhombomeres) (Figure 1D). The anterior and posterior-most crypto-rhombomeres define the borders between the hindbrain and the midbrain and spinal-cord (Marin et al., 1995; Cambronero and Puelles, 2000; Marín et al., 2008; Puelles et al., 2013; Soares et al., 2013; Tomás-Roca et al., 2016; Watson et al., 2017).

In all vertebrates, hindbrain compartmentalization is controlled by combinatorial expression and activity of multiple families of transcription and signaling factors across each rhombomere, leading to the generation of distinct cell lineage compartments (Kiecker and Lumsden, 2005; Weisinger et al., 2008, 2010; Chambers et al., 2009; Kayam et al., 2013; Frank and Sela-Donenfeld, 2018; Parker and Krumlauf, 2020). The identity of individual rhombomeres instructs the neuronal differentiation plan in the hindbrain which is manifested in the migration of neurons, axons, and neural crest cells and the generation of different motor or sensory nuclei along defined dorsoventral (DV) and AP positions (Lumsden and Keynes, 1989; Marín and Puelles, 1995; Cambronero and Puelles, 2000; Trainor and Krumlauf, 2001; Briscoe and Wilkinson, 2004; Guthrie, 2007; Marín et al., 2008; Narita and Rijli, 2009; Puelles et al., 2013; Tomás-Roca et al., 2016; Di Bonito and Studer, 2017). Based on the pioneer insights of Wilhelm His from the 19th century, the dorsolateral margin of the longitudinal hindbrain has been defined as the rhombic lip (RL), which has been found to serve as a source of a number of hindbrain neuron populations that are generated through tangential migrations of neuroblasts which delaminate from the RL (Glover et al., 2018). These different neuronal subtypes were found to depend on their rhombomeric origin; in $\mathrm{r} 1$, the most dorsal part of the RL contributes a large migratory cell population that forms the external and internal granular layers of the cerebellum (Ben-Arie et al., 1997; Wingate and Hatten, 1999; Köster and Fraser, 2001; Machold and Fishell, 2005). In r2-r6, the same RL domain generates auditory and vestibular nuclei, through which information is processed and relayed to the upper brain and spinal cord, whereas, in r6-r8, it will give rise to multiple pre-cerebellar nuclei which relay peripheral sensation to the cerebellum through mossy fiber neurons (Altman and Bayer, 1980, 1987a,b,c,d; Rubel and Parks, 1988; Cambronero and Puelles, 2000; Rodriguez and Dymecki, 2000; Bermingham et al., 2001; Díaz et al., 2003; Ryugo and Parks, 2003; Pasqualetti et al., 2007; Hoshino et al., 2013; Kratochwil et al., 2017; Díaz and Puelles, 2019; Elliott et al., 2021). Similarly, different types of respiratory and viscerosensory nuclei are suggested to be born from more ventral positions of the RL at distinct axial levels, such as the parabrachial and KöllikerFuse nuclei that derive from $\mathrm{r} 1$, the A5 and intertrigeminal region that derives from $\mathrm{r} 4-\mathrm{r} 6$, the PreBötzinger complex and retrotrapezoid nucleus (RTN) that derive from $\mathrm{r} 3 / \mathrm{r} 5$, and the nucleus tractus solitaries that is thought to derive from more posterior rhombomeres (Qian et al., 2001; Gray, 2008). The hindbrain is also divided along its DV axis into a basal and alar plate, at which discrete neuronal progenitors become specified 

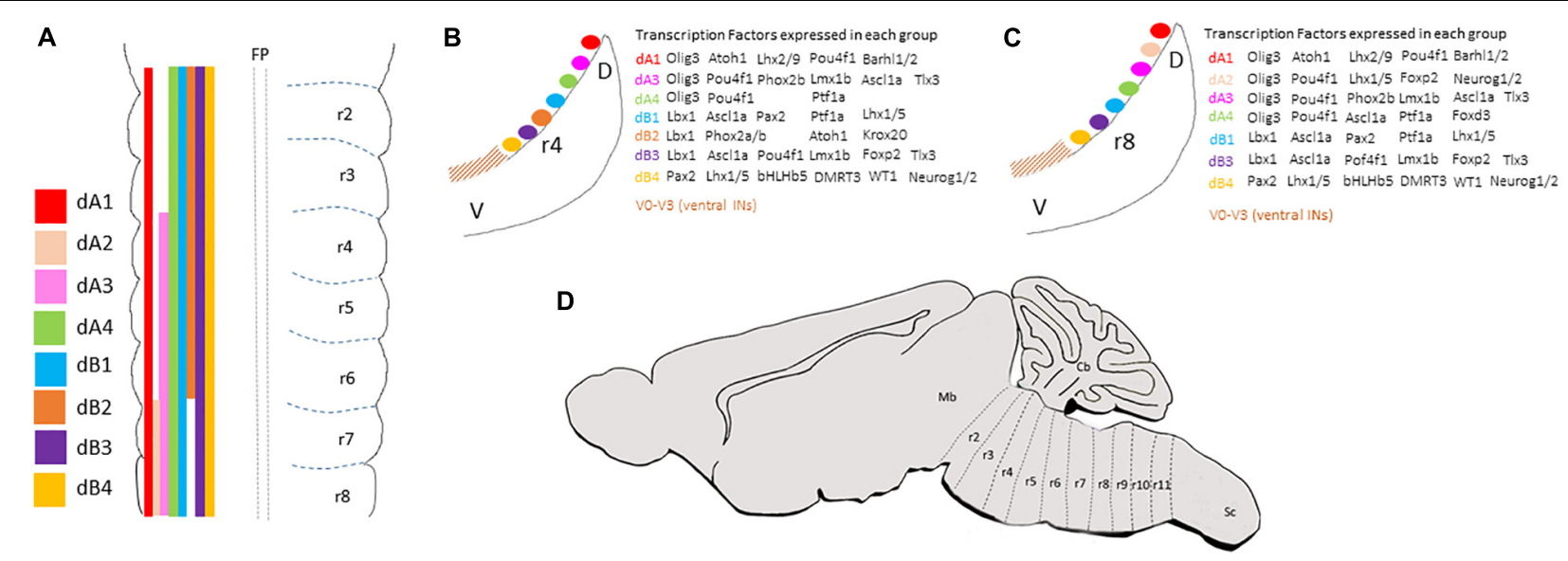

FIGURE 1 | (A) Summary of the anterior-posterior distribution of dA and dB subclasses along the overt hindbrain rhombomeres (as classified in Lumsden and Krumlauf, 1996). (B,C) Schematic transverse sections taken from rhombomere 4 (B) or rhombomere 8 (C) levels of E3 chick embryo to show the dorsal-ventral distribution of $\mathrm{dA}$ and $\mathrm{dB}$ subclasses and the combination of transcription factors expressed in each subclass. (D) A schematic sagittal section representing the classification of hindbrain segments into 12 rhombomeres based on fate map analyses (as classified in Marín et al., 2008; Tomás-Roca et al., 2016). Cb, cerebellum; $\mathrm{D}$, dorsal; V, ventral; Mb, midbrain; r, rhombomere; Sc, spinal cord.

and differentiate in distinct longitudinal DV locations that are uniform along with the hindbrain (Figures 1A-C) (Marin et al., 1995; Shoji et al., 1996; Cambronero and Puelles, 2000; Schubert et al., 2001; Wang et al., 2005; Zijing et al., 2008; Storm et al., 2009; Hernandez-Miranda et al., 2017). The neural diversity along the AP and DV axes is critical for the correct elaboration of functional circuits that shape the adult brainstem.

Investigation of the neuronal patterns along the dorsal hindbrain has identified 6-8 progenitor domains that are born in designated positions in some or all rhombomeres (Figure 1A). In contrast, it is not yet clear whether the same set of progenitor domains exist at the rostral-most hindbrain levels ( $\mathrm{r} 0$ and $\mathrm{r} 1$ ), which are largely patterned by the isthmic organizer. Notably, their precise DV distributions continue further caudally to the spinal cord. Altogether, these progenitors will give rise to second-order interneurons that act as first central relay stations for sensory-motor connections, which intervene in reflex arcs or are largely conveyed from the spinal cord and PNS to upper brain centers or to the spinal cord (Logan et al., 1998; Maklad and Fritzsch, 2003; Ryugo and Parks, 2003; Landsberg et al., 2005; Sieber et al., 2007; Rose et al., 2009a,b; Storm et al., 2009). These hindbrain dorsal interneurons (termed here dINs) are divided into class $A(\mathrm{dA})$ and $B(\mathrm{~dB})$ neurons based on their DV positions; those who arise in the dorsal microzones of the hindbrain are classified as class A and express the bHLH transcription factor Olig3, while those born more ventrally in the alar plate and express the Ladybird Homeobox transcription factor Lbx1 are referred as class B (Figure 1A) (Gross et al., 2002; Müller et al., 2002, 2005; Zijing et al., 2008; Hoshino, 2012; Puelles, 2013). Each of the two groups is further subdivided along the DV axis based on the expression of unique sets of specification and differentiation genes in various neuroepithelial microzones, that also differ along the ventricular/mantle zone, reflecting the differential progenitor origin and molecular profile of each neuron (Figures 1B,C)
(Wang et al., 2005; Fujiyama et al., 2009; Kohl et al., 2012; Gray, 2013; Hernandez-Miranda et al., 2017).

The specification of the different $\mathrm{dA} / \mathrm{dB}$ subclasses requires coordinated signaling cues that arise from the roof and floor plate (FP) (i.e., BMP, Wnt, and $\mathrm{SHH}$ ), and provide positional information which leads to the birth of individual neuronal fates (Liem et al., 1997; Lee et al., 1998, 2000; Briscoe et al., 1999; Gaufo et al., 2000; Vogel-Höpker and Rohrer, 2002; Müller et al., 2005; Storm et al., 2009; Tilleman et al., 2010; MorenoBravo et al., 2014; Lihua et al., 2019). The mechanisms by which these morphogens act to pattern hindbrain dINs are not fully understood and will not be discussed further in this review.

Following the differentiation of neurons, they start migrating to their final destinations in the mantle layer as well as to project axons that extend toward their target sites in a stepwise manner, under the control of guidance cues along their pathways (TessierLavigne and Goodman, 1996; Chédotal and Richards, 2010). In the developing hindbrain, axonal growth initiates at particular DV positions within each rhombomere and projects into defined commissural and ipsilateral tracts. Upon the completion of the axonal circuit, these tracts will project sensory information from the periphery, spinal cord, and brainstem to higher brain centers, as well as transmit motor commands from the brain to the spinal cord (Rubel and Parks, 1975, 1988; Díaz et al., 1998; Howell et al., 2007; Renier et al., 2010; Kalinovsky et al., 2011; Di Bonito et al., 2017). Over the past 50 years, classical labeling techniques have thoroughly mapped multiple axonal tracts in the hindbrain. Yet, their association to specific $\mathrm{dA} / \mathrm{dB}$ sub-populations was missing, as most of these studies preceded the development of genetic tools to fate map individual cell groups. Subsequently, the contribution of different $\mathrm{dA} / \mathrm{dB}$ neural precursors into neuronal populations of different brainstem nuclei has begun to be recognized, as well as the identification of genetically identified tracts that emerge from these centers. Nevertheless, the fate and axonal routes of some of these 
subgroups are not fully revealed, nor was the delineation of the entire axonogenesis of individual $\mathrm{dA} / \mathrm{dB}$ subpopulations, from soon after their differentiation until their axons terminate at their targets.

This review aims to cover the gap between the vast knowledge on hindbrain nuclei projections and their association to specific $\mathrm{dA} / \mathrm{dB}$ INs along the hindbrain AP axis. We will not discuss $\mathrm{r} 0$, $\mathrm{r} 1$, and their contribution to the isthmus and cerebellum, and mainly concentrate on $\mathrm{r} 2-\mathrm{r} 7 / 8$, the rhombomeric units which are morphologically evident and coincide in the two rhombomereclassification systems in use (Lumsden and Krumlauf, 1996; Tomás-Roca et al., 2016). Moreover, past and current knowledge regarding molecular cues that govern the axonal growth of $\mathrm{dA} / \mathrm{dB}$ neurons will also be presented.

\section{Class A: dA1 Neurons Specification and Fate}

This neuronal subpopulation is positioned in the dorsal most portion of the hindbrain RL, bordering the expanded roof plate of the fourth ventricle (Figure 1A). The specification of this excitatory/glutamatergic neuronal population is dependent on the co-expression of Olig3 and the basic helix-loop-helix (bHLH) transcription factor Atonal homolog 1 (Atoh1) in the ventricular zone. A combinatorial expression of the LIM-homeodomain (LIM-HD) transcription factors Lhx2 and Lhx9, the Barh like homeobox transcription factor Barhl1/2, and the POU domain class 4 transcription factor 1 Pou4f1 accompanies dA1 neural differentiation and migration (Figures 1B,C) (Helms and Johnson, 2003; Wang et al., 2005; Kohl et al., 2012; HernandezMiranda et al., 2017). Moreover, the two Lim homeobox transcription factors, Lmxla and Lmxlb, which are expressed in the roof-plate dorsal to dA1 group, are necessary for their specification (Elliott et al., 2021). Extensive fate map studies in mouse and chick hindbrains, together with the generation of knock-in and knock-out mice lines revealed a wealth of derivatives that originate from $\mathrm{dA} 1$ INs, depending upon their rhombomeric origin and time of birth; At r1, dA1 neurons give rise to cerebellar granule cells whereas at $\mathrm{r} 2-\mathrm{r} 8$ they give rise to excitatory neurons that assemble various sorts of brainstem nuclei, with some divergence in their rhombomeric origin in avian and mammalians (Farago et al., 2006; Nothwang, 2016; Lipovsek and Wingate, 2018). These include Atoh $1^{+}$neurons in several nuclei subtypes in the auditory system, such as in the ventral and dorsal cochlear nuclei (VCN, DCN) and the superior olivary nuclei (SON), Atoh $1^{+}$neurons in the spinal, medial, and lateral vestibular nuclei (Sp5, MVN, and LVN) and in the vestibular nucleus $\mathrm{X}$, Atoh $1^{+}$neurons in the main and descending sensory spinal trigeminal nuclei and Atoh $1^{+}$ neurons which establish the various precerebellar nuclei (PCNs), which include the pontine gray nuclei (PGN), reticulotegmental nucleus, lateral reticular nucleus (LRN), and external cuneate nucleus (ECN) (Ben-Arie et al., 1997; Bermingham et al., 2001; Landsberg et al., 2005; Machold and Fishell, 2005; Wang et al., 2005, 2020; Farago et al., 2006; Kawauchi et al., 2006; Zijing et al., 2008; Maricich et al., 2009; Ray and Dymecki, 2009; Rose et al., 2009a,b; Machold et al., 2011; Fu et al., 2013; Hoshino et al., 2013;
Lipovsek and Wingate, 2018; Elliott et al., 2021). Interestingly, the specification of the choroid plexus is also dependent on the presence of the adjacent dA1 neurons (Elliott et al., 2021). All of the above-mentioned nuclei centers fail to be generated normally in Atoh1-null mice, as well as in mice lacking the roof-plate LimHD proteins Lmx1A/B, which show various neurodevelopmental defects and die at birth (Mishima et al., 2009; Rose et al., 2009a,b; van der Heijden and Zoghbi, 2018; Chizhikov et al., 2021). Despite this extensive knowledge, the mechanisms that drive $\mathrm{dA} 1 /$ Atoh $^{+}$dINs to give rise to such a remarkable wealth of neuronal fates are only partially clear.

\section{dA1 Axonal Projections}

Classical axonal labeling approaches in the chick embryonic hindbrain uncovered multiple ascending and descending tracts that arose from ipsilateral or contralateral neurons (Lumsden and Keynes, 1989; Marín and Puelles, 1995; Díaz et al., 2003; Guthrie, 2007). For example, DiI labeling of caudal dorsal hindbrain commissures identified them as formed by secondorder vestibular neurons (cC-VC), that project axons which turn into the Dorsal Funiculus (DF) (Díaz et al., 1998; Zhu et al., 2006). Tracing the projections of the cochlear nuclei (CN) showed that the mammalian VCN, or its avian homolog nucleus magnocellularis (NM), project ipsi and contralateral axons to the mammalian SON/avian nucleus laminaris (NL). This symmetrical connectivity results in bilateral excitatory input to the Medial Superior Olivary (MSO) center in the SON in mammalians, or to the NL in avians, that in turn project through ipsi and contralateral lateral lemniscus to midbrain auditory centers (Rubel and Parks, 1988; Kil et al., 1995; Moore, 2000; Carr and Soares, 2002; Seidl et al., 2013). Moreover, labeling of the caudal rodent brainstem revealed ipsi and contralateral projections from different PCN to the cerebellum via Mossy Fiber (MF) tracts (Gerrits et al., 1984; Altman and Bayer, 1987a,c,d; Bourrat and Sotelo, 1990; Cicirata et al., 2005; Okada et al., 2007). Furthermore, labeling of excitatory axons of the MVN demonstrated their projections to other brainstem vestibular nuclei together with their projections to the cerebellum as mossy fibers (Ando et al., 2020). Based on the neuronal cell body positions of these axons, it is likely that most of those tracts originate from dA1 neurons at the dorsal RL (excluding Atoh $1^{+}$ neurons in various $\mathrm{VN}$ as their axonal projections and later fates are still vague). However, only with the development of genetic tools that utilized Atho1-enhancer elements to label neuronal precursors in the dorsal RL, these trajectories could be attributed to the dA1 subpopulation (Helms and Johnson, 1998; Wang et al., 2005; Farago et al., 2006; Okada et al., 2007; DiPietrantonio and Dymecki, 2009; Rose et al., 2009a,b; Kohl et al., 2012). Subtractive fate map approaches that combined targeting of Atho $1^{+}$neurons under rhombomere-specific regulatory elements further enabled to reveal the rhombomeric origin of various dA1-derived nuclei (Farago et al., 2006; Maricich et al., 2009; Lipovsek and Wingate, 2018). As such, dA1/Atoh $1^{+}$neurons that emerge from r2r5 in mammalians or r5-r8 in avian, and contribute excitatory neurons to the mammalian VCN/avian NM were found to extend ipsilateral and contralateral projections to the mammalian MSO/avian NL (Figure 2C), while those arising from r6-r8 give 

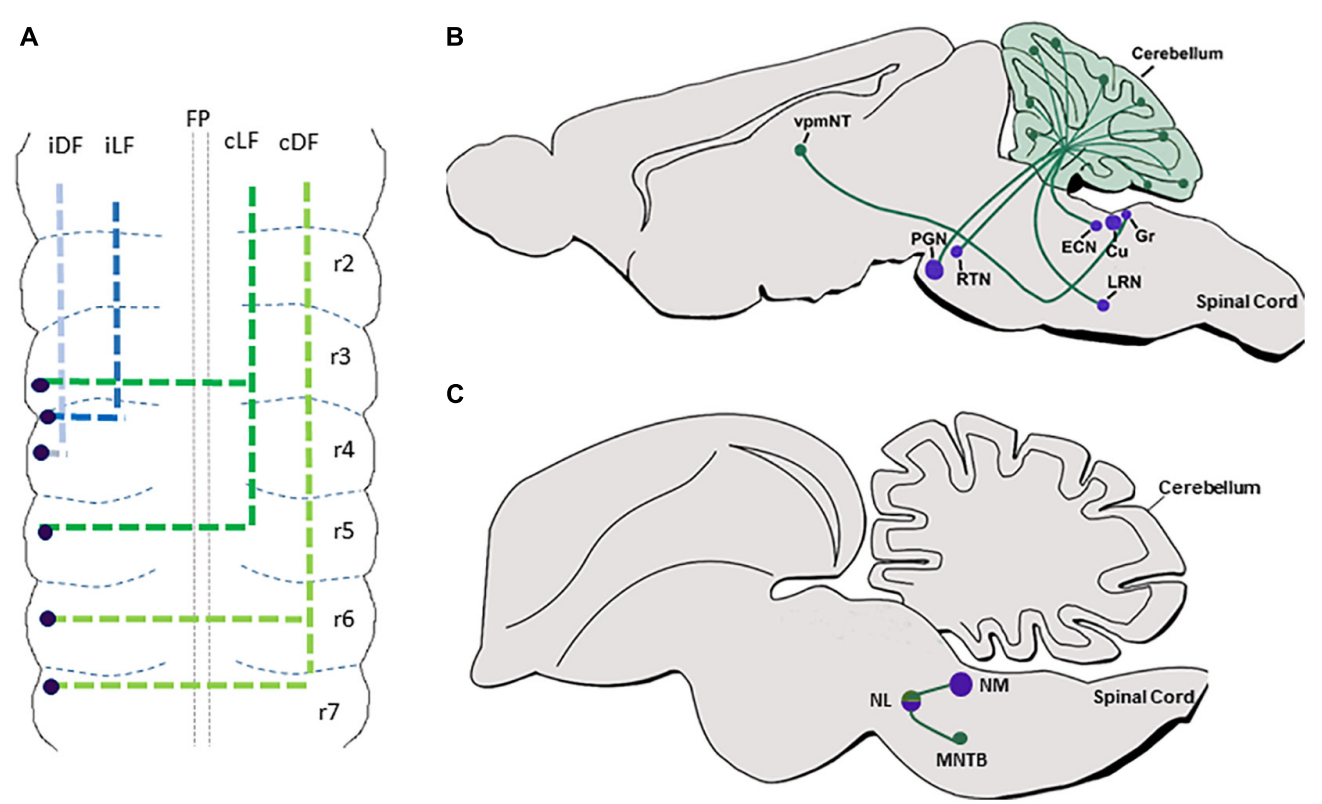

FIGURE 2 | dA1 axonal projections. (A) A summary of dA1 axonal projections as seen in a flat-mount view of E6.5 chick embryonic hindbrain. dA1 cell bodies are shown in blue dots. Each axonal tract is shown in a different color and refers to a distinct funiculus. (B) A schematic sagittal section of the main pre-cerebellar axonal circuits of the dA1 subclass. Different dA1-derived pre-cerebellar nuclei (purple circles) are shown to project axons (green lines) to the cerebellar granular layer or the thalamus (green circles). (C) A schematic sagittal section of the main auditory axonal circuit of the dA1 subclass in the chick hindbrain. $\mathrm{dA1}$-derived NM and NL centers (purple circles) are shown to project axons (green lines) to local auditory nuclei (green circles). FP, floor plate; iDF, ipsi dorsal funiculus; iLF, ipsilateral funiculus; CLF, contralateral lateral funiculus; cDF, contralateral dorsal funiculus; PGN, pontine gray nucleus; RTN, reticulotegmental nucleus; LRN, lateral reticular nucleus; ECN, external cuneate nucleus; Cu, cuneate; Gr, gracile; vpmNT, the ventral posteromedial nucleus of the thalamus; NM, nucleus magnocellularis; NL, nucleus laminaris; MNTB, medial nucleus of the trapezoid body.

rise to multiple PCN and extend ipsi and contralateral axons to the granular layer of the cerebellum as MFs (Figure 2B).

Despite this extensive knowledge, data regarding the precise en-route axonal patterns of individual dA1-neuronal subgroups was limited, mostly since dA1-derived axons were largely traced at stages when their soma have already settled at their final nuclear destination. Moreover, since germ-line transgenesis resulted in the labeling of all Atoh $1^{+}$lineages on both sides of the hindbrain, the ability to trace unilateral projections at subsequent stages was compromised. In a series of studies in the chick hindbrain, we aimed at tracing the sequential axonal growth of dA1 INs at multiple stages, using unilateral electroporation of plasmids encoding dA1-specific enhancers upstream of Cre recombinase together with nuclear, cytoplasmic, or synaptic GFP reporters. Hindbrains were electroporated at stages when $\mathrm{dA} 1 /$ Atoh $^{+}$cells are specified and their axons were traced for 2-16 days (Figure 2A) (Kohl et al., 2012, 2013; Hadas et al., 2014). Axons were found to project into the ipsi and contralateral DF and lateral funiculus (LF). Yet, axons that originated from r6-r7 were found to project along with the DF toward the cerebellum and midbrain, whereas those originating from $\mathrm{r} 3-\mathrm{r} 5$ ascended in the LF in a less-tight bundle toward the midbrain (Figure 2A). Pre-synaptic connections of axons from r6-r7 levels terminated in the cerebellar outer and inner granular layers (Figure 2B) (Kohl et al., 2012). These results expanded previous studies performed on post-natal pre-cerebellar-cerebellar mossy fiber circuitry (Marín and Puelles, 1995; Díaz et al., 2003;
Wang et al., 2005; Fujiyama et al., 2009; Rose et al., 2009a,b; Kohl et al., 2012, 2013, 2015), by tracing the gradual assembly of these circuits (Figure 2). Using this approach, we have also recently decoded the gradual axonal circuit formation of the binaural auditory system. In this system, dA1 neurons from the avian NM/mammalian anteroventral cochlear nucleus (AVCN) receive temporally locked excitation from the auditory nerve, and in turn, send bilaterally segregated signals to the avian NL/mammalian medial superior olive (MSO), (Cramer et al., 2000; Ryugo and Parks, 2003). The bipolar neurons in the NL/MSO are specialized to compute interaural time differences which is critical for sound localization and segregation (Overholt et al., 1992; Nothwang, 2016). Although the anatomy and physiology of this circuitry are well known, its stage-bystage assembly was obscure. dA1-specific reporter plasmids were introduced into $\mathrm{dA} 1 / \mathrm{NM}$ precursors at $\mathrm{r} 5$ and their axons could be traced to gradually exhibit the characterized pattern of NMNL projection (Figure 2C). This selective dissection allowed us to demonstrate a dA1 subtype-specific, longitudinal assessment of axonal events throughout hindbrain development, from E2.5 to E19 (Wang et al., 2020).

\section{Class A: dA2 Neurons Specification and Fate}

dA2 subpopulation, which appears ventral to dA1 group, exists only from $\mathrm{r} 7$ and caudally (Figure 1A). This excitatory subgroup 
is derived of Olig $3^{+} / \mathrm{Neurogenin} 1 / 2^{+}\left(\right.$Neurog $\left.1 / 2^{+}\right)$progenitors that upon maturation express the transcription factors Lhx1/5, Forkhead box protein 2 (Foxp2), and Pou4f1 (Figure 1C) (Landsberg et al., 2005; Storm et al., 2009). Notably, although this subgroup extends along the spinal cord as dI2, their express Foxd3 instead of FoxP2 (Storm et al., 2009). The mechanisms underlying this molecular difference as well as the appearance of dA2 INs only in the posterior RL, are currently unknown. Utilization of Wnt1-reporter mouse to lineage-trace RL-cell populations demonstrated a small contribution of $\mathrm{r} 7$-derived Neurog $1^{+}$neurons to the inferior olivary nucleus (ION), a major pre-cerebellar center that connects to the cerebellar Purkinje layer via climbing fiber (CF) axons (Landsberg et al., 2005). Yet, further fate map analyses are required to fully confirm dA2 fate as part of the ION, as dA4 subgroups were also suggested to assemble the ION, as will be described below.

\section{dA2-Axonal Projections}

The axonal projection patterns of $\mathrm{dA} 2$ neurons have not yet been delineated. This is in contrast to the spinal cord, where genetic labeling of $\mathrm{dI} 2$ INs in the chick spinal cord uncovered their contralateral ascending or descending projections, according to the thoracic/sacral level, before turning laterally in the white matter toward the LF (Avraham et al., 2009). Genetic lineage tracing of dA2 INs is needed to trace their axons and decipher whether they join the CF tract as well as possible other tracts in the developing brainstem.

\section{Class A: dA3 Interneurons Specification and Fate}

The dA3 subpopulation originates ventrally to the dA1 subgroup in r4-r7 (Figure 1A). Similar to other excitatory class A dINs, dA3 progenitors express Olig3 and Pou4f1, in addition to the Paired-like homeobox 2b (Phox2b), T-Cell Leukemia Homeobox 3 (Tlx3), and LIM homeobox protein 1-beta (Lmx1b). Moreover, they are the most dorsal subgroup that expresses the mammalian achaete-scute family bHLH transcription factor $1\left(\mathrm{Ascl}^{+}\right)$ (Figures 1B,C) (Qian et al., 2001; Sieber et al., 2007; Kim et al., 2008; Storm et al., 2009). Previous studies have suggested the role of Tlx3 in regulating the dA3-glutamatergic cell fate (Cheng et al., 2005; Chen et al., 2012). However, Tlx activity cannot be attributed to all excitatory $\mathrm{dA} / \mathrm{dB}$, as it is not expressed in other glutamatergic subgroups.

dA3 INs were found to contribute to various viscerosensory autonomic components in the hindbrain (Brunet and Pattyn, 2002; Qian et al., 2002; Dauger et al., 2003; D’Autréaux et al., 2011; Gray, 2013). dA3 (Phox $2 b^{+} / \mathrm{Tlx}^{+} / \mathrm{Ascl}^{+}$) neurons that emerge from $r 4-r 7$ contribute to the nucleus of the solitary tract (NTS), a major relay station for visceral sensory information regulating the activity of the cardiovascular, respiratory, vocalization, and digestive systems (Qian et al., 2001; Dauger et al., 2003; Pattyn et al., 2006; Sieber et al., 2007; Storm et al., 2009; Hernandez-Miranda et al., 2017; Gasparini et al., 2020). In addition, dA3 neurons deriving from r7-r8 contribute to the area postrema (AP) nucleus, a chemoreceptive center in the dorsal hindbrain that responds to toxins via chemically induced vomiting, as well as to A5/A7 noradrenergic clusters of the lateral tegmental area of the pons, which are suggested to be involved in vasomotor and respiratory activities, as well as in transmitting noradrenergic inputs to the spinal cord. Finally, dA3 INs were also suggested to give rise to the non-tyrosine hydroxylase expressing neurons of the intermediate reticular formation at the rostral medullary levels, implicating their additional involvement in secondary viscerosensory processing (Anderson et al., 1997; Qian et al., 2001; Kang et al., 2007; Gray, 2013). Interestingly, Phox $2 b$ is indispensable for the viscerosensory fate of $\mathrm{dA} 3$, since in its absence visceral sensory neurons resemble $\mathrm{dB} 3$ somatic sensory neurons, which express Tlx3 and Ascl1 (like dA3) but are devoid of Phox2b (D’Autréaux et al., 2011).

\section{dA3 Axonal Projections}

In concordance with their homing at different autonomic centers, dA3-INs extend multiple axonal trajectories (Figure 3). Most studies traced projections of mature nuclei in adult brains. For example, projections from the NTS were examined in adult rodent brains by multiple antero/retrograde labeling approaches, revealing complex projections that ascend in ipsi/contralateral ventral/dorsal paths to innervate different sub-nuclear sites in the parabrachial nucleus $(\mathrm{PBN}), \mathrm{RTN}$, rostral ventrolateral medullary nucleus as well as within the respiratory compartments of the rostroventral respiratory group, preBötC and BötC nuclei (Herbert et al., 1990; Williams et al., 1996; Cunningham and Sawchenko, 2000; Karimnamazi et al., 2002; Alheid et al., 2011; Fu et al., 2019). Recently, a subpopulation of aldosterone-sensitive neurons, which express the dA3 markers Phox2B/Lmx1b, were also found to localize in the NTS and to control sodium appetite by projecting anteriorly to the PBN and pre-locus coeruleus ( $\mathrm{pLC}$ ) complex in the prepontine hindbrain ( $\mathrm{r} 1)$ as well as to the bed nucleus of the stria terminalis in the forebrain (Gasparini et al., 2018). Other NTS axons project ventrally and caudally to converge into the anterolateral funiculus toward different segments of the cervical and thoracic spinal cord, where axonal processes diverge and enter the ventral horn to innervate pre-motor neurons (Norgren, 1978; Mtui et al., 1993). Another ipsilateral projection elongates from the NTS through the reticular formation to terminate at the facial motor nucleus (Norgren, 1978). Finally, the NTS projects bilaterally to the hypoglossal nucleus, by crossing the midline and extending over the dorsomedial reticular formation toward the hypoglossal nucleus (Norgren, 1978). Notably, although advanced combinations of axonal labeling in knockout or reporter mice lines enabled to link Phox $2 \mathrm{~b}^{+}$neurons in the NTS with these circuits (Qian et al., 2001; Dauger et al., 2003; Pattyn et al., 2006; Sieber et al., 2007; Smith et al., 2009; Storm et al., 2009; Hernandez-Miranda et al., 2017), tracking the gradual axonal growth of distinct dA3 INs before and after populating different subdomains along the NTS is missing. Unraveling this issue is particularly important since the NTS contains diverse intermingled subpopulations of neurons that modulate distinct functions by their extensive projections.

Anterograde axonal and retrograde cell body tract-tracing methods in the AP nucleus of the adult rat demonstrated its connectivity to a variety of different nuclei in both the medulla and upper brain regions. AP projections target the adjacent 


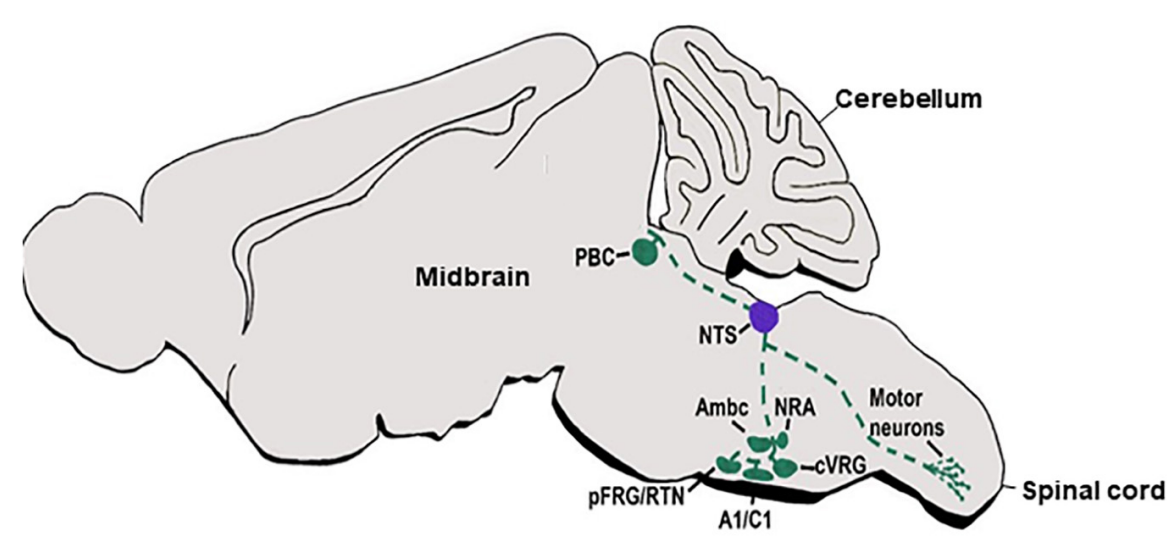

FIGURE 3 | dA3 axonal projections. A schematic sagittal section of a main viscerosensory autonomic axonal circuit of the dA3 subclass. dA3-derived NTS nuclei (purple circle) are shown to project multiple axonal trajectories (green broken lines) to various target sites in the hindbrain or spinal cord (green circles). NTS, nucleus tractus solitaries; Pfrg/RTN, parafacial respiratory group/reticulotegmental nucleus; Ambc, ambiguous nucleus, compact part; cVRG, caudal ventral respiratory group; NRA, nucleus retro ambiguous; PBC, parabrachial.

NTS and the PBN, as well as the ambiguous (AMB) nucleus, the dorsal motor nucleus of the vagus, dorsal regions of the tegmental nucleus, the cerebellar vermis, the paratrigeminal nucleus, the ventrolateral catecholaminergic column in the medulla, and the spinal trigeminal tract (van der Kooy and Koda, 1983; Shapiro and Miselis, 1985; Price et al., 2008; Stein and Loewy, 2010). Complex axonal trajectories extend from the AP to target these nuclei sites. For instance, ipsilateral AP axons extend either caudally or rostrally to target the NTS, or project in a rostroventral direction around the solitary tract and turn ventrolaterally via the reticular formation to either target the AMB or to further bifurcate and extend dorsorostrally toward the PBN and the pLC. Another AP tract joins the ventral spinocerebellar tract to enter the cerebellum, or projects dorsorostromedially and divides further to either terminate at the PBN or elongate further and terminate at the mesencephalic trigeminal nucleus (Shapiro and Miselis, 1985). Notably, no data exist regarding the development of these tracts in the embryo.

Retrograde and anterograde axonal tracing of the noradrenergic A5/A7 clusters in the adult rodent brain has demonstrated innervations to multiple midbrain regions of dopamine neurons (i.e., retrorubral field, ventral tegmental area, substantia nigra, interfascicular nucleus, ipsilateral rostral/central linear, and nuclei) (Mejías-Aponte et al., 2009). The A5 cell group was also found to project to the central nucleus of the amygdala, perifornical and dorsal areas of the hypothalamus, paraventricular nucleus of the thalamus, and the bed nucleus of the stria terminalis. This cluster also projects to PBN, the NTS, and the ventrolateral reticular formation of the medulla (Byrum and Guyenet, 1987). Further traditional or genetic axonal-labeling approaches had enabled the tracing of spinal projections of A5/A7 nuclei in adult rodent or cat brains, revealing descending projections via the ipsi/contralateral ventral and lateral funiculi. These projections further branch in a complementary fashion to reach the dorsal/ventral horns of the spinal cord at different axial levels (Fritschy and Grzanna, 1990; Clark and Proudfit, 1993; Bruinstroop et al., 2012). Finally, the non-tyrosine hydroxylase expressing neurons of the intermediate reticular formation in the medulla were suggested to relay cortical input to gustatory centers at the NTS and the PBN (Kang et al., 2007; Gray, 2013).

Altogether, dA3 INs exhibit complex fates and display multiple ascending and descending axonal routes (Figure 3), emphasizing their important contribution to various autonomic circuits in the CNS. Yet, knowledge is still missing regarding the developmental mechanisms that drive the different fates of $\mathrm{dA} 3$ subpopulations in individual rhombomeres. Further genetic-lineage tracing experiments of dA3 INs are also in need to fully determine the precise contribution of dA3 INs to the extensive axonal tracts described above Notably, one of the dA3 markers, Lmx1b, was recently shown to be necessary for controlling axonal growth of serotonergic 5-HT neurons in the hindbrain and dopaminergic circuits in the midbrain (Chabrat et al., 2017; Donovan et al., 2019), raising the possibility that Lmx1b may also affect dA3 axonal growth decisions.

\section{Class A: dA4 Interneurons Specification and Fate}

The dA4 population is the most ventral one amongst class A dINs (Figure 1A). Fate mapping experiments in chick and mice revealed that they derive from Pou $41^{+} / \mathrm{Olig}^{+} / \mathrm{Mash}^{+}$ progenitors that also express the bHLH factor Pancreas Specific Transcription factor 1a (Ptfla) (Figures 1B,C) (Fedtsova and Turner, 1995; Sieber et al., 2007; Yamada et al., 2007; Storm et al., 2009; Hidalgo-Sánchez et al., 2012). dA4 neuronal group is the only excitatory subgroup that expresses Ptfla (Yamada et al., 2007; Storm et al., 2009; Gray, 2013), like all other $\mathrm{Ptfl}^{+}$dINs in the hindbrain or spinal cord are inhibitory (GABAergic/Glycinergic) and were suggested to depend on Ptf1a for their inhibitory neuronal fate (Glasgow et al., 2005; Hoshino et al., 2005). While the molecular profile of dA4 cells is uniform from $\mathrm{r} 2$ to $\mathrm{r} 6$, in $\mathrm{r} 7$ this subgroup also expresses Foxd3 (Sieber et al., 2007; Yamada et al., 2007; Storm et al., 2009; 
Iskusnykh et al., 2016). So far, the fate of dA4 neurons from $\mathrm{r} 2-\mathrm{r} 6$ is not clear. At variance, dA4 precursors originating from r7 to r11 were suggested to contribute to the ION, which is positioned in the caudal-ventral brainstem and coordinates signals to and from the cerebellar Purkinje cell layer to regulate motor coordination and learning (Sieber et al., 2007; Yamada et al., 2007; Zijing et al., 2008; Storm et al., 2009; Iskusnykh et al., 2016; Watson et al., 2019). Formation of ION is missing in Ptf1a-null zebrafish or mouse embryos, where Ptf1a ${ }^{-/}$cells shifted their fate to become MF neurons (Yamada et al., 2007; Itoh et al., 2020). Intriguingly, as Ptfla or Ascl1 proteins are not only expressed in dA4 neurons but also their flanking $\mathrm{dB} 1 / \mathrm{dA} 3$ subpopulations, further enhancer-intersection based approaches are required to distinctly map dA4 precursors at different AP levels, rather than their co-labeling with neighboring neuronal groups.

\section{Axonal Projection Patterns}

Climbing fiber axons were traced by traditional retrograde/anterograde approaches in all vertebrates and found to originate from the caudal and rostral ION (Altman and Bayer, 1987b; Paradies and Eisenman, 1993; Sawada et al., 2008;
Reeber et al., 2013). While all ION axons cross the FP and grow in the dorsomedial inferior cerebellar peduncle toward the contralateral cerebellum, those originating from the caudal ION, which lies in crypto-rhombomeres r10, r11, project to the posterior cerebellum whereas those deriving from more rostral ION position ( $\mathrm{r} 8$ and $\mathrm{r} 9$ ) enter the cerebellum through the lateral inferior cerebellar peduncle, also termed the restiform body, turn to a dorsolateral route and innervate the lower strata of the embryonic Purkinje cell multilayer. Fate map studies of $\mathrm{Ptfla}^{+}$neurons in wild type and mutated zebrafish/mouse embryos confirmed that Ptfla ${ }^{+}$ION neurons extend excitatory commissural projections that innervate Purkinje cells (Figure 4A) (Yamada et al., 2007; Bae et al., 2009; Hashimoto and Hibi, 2012; Itoh et al., 2020). However, a direct link between projections of lineage-traced dA4 neurons from different ION subdomains to different cerebellar lobules is still missing. Moreover, as most studies traced this circuit at stages following ION formation, the gradual growth of $\mathrm{dB} 4 / \mathrm{Ptfla}^{+}$axons from soon after their differentiation in the $\mathrm{RL}$ remained elusive. Using an enhancer-based conditional expression system in the chick embryo combined with the Ptfla enhancer element, we have targeted $\mathrm{PTF}^{+}{ }^{+}$precursors

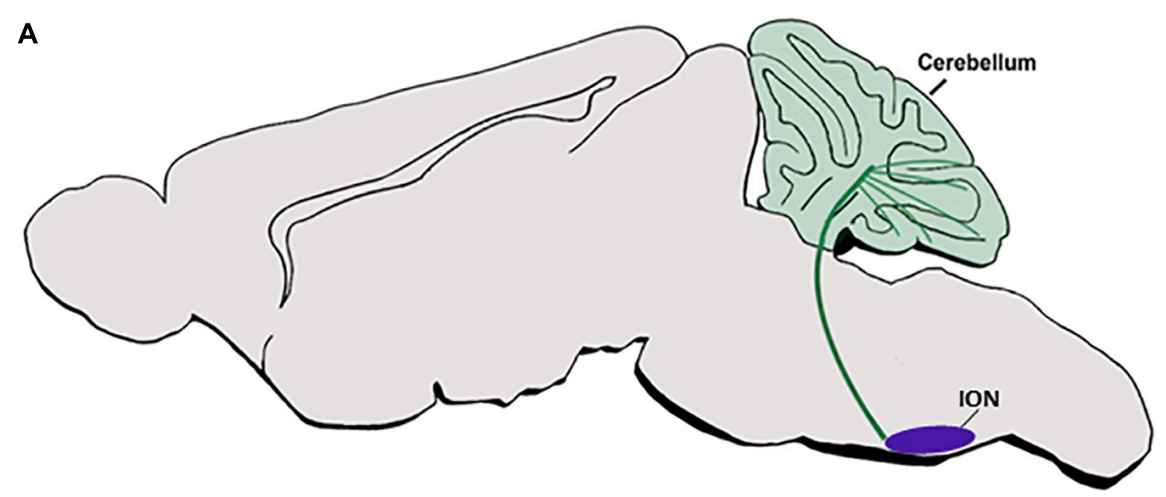

B

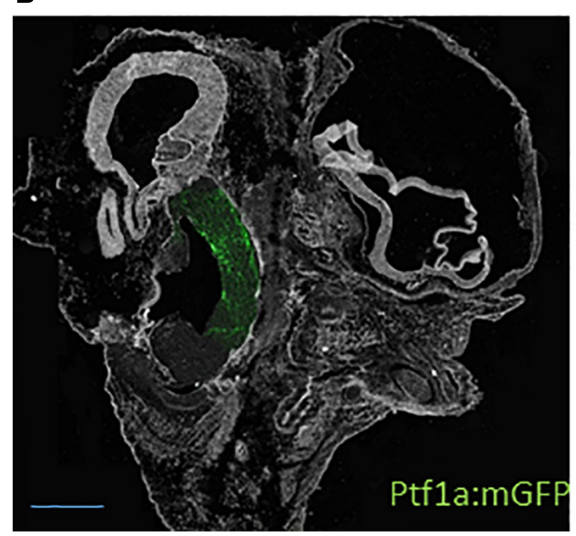

C

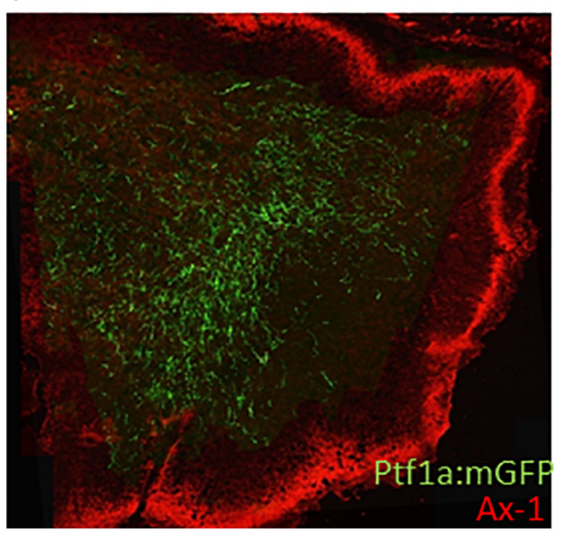

FIGURE 4 | dA4 axonal projections. (A) A schematic sagittal section of the climbing fiber (CF) circuit of the dA4 subclass. dA4-derived ION (purple circle) is shown to project axonal trajectory (green line) to the Purkinje cell layer of the cerebellum (green circles). (B,C) Sagital sections from E9.5 (B) or E13.5 (C) chick embryos that were electroporated at E2.5 with a Ptf1a enhancer:Cre based plasmid together with lox-membranal GFP-lox plasmid (ptf1a:mGFP) to specifically label dA4 neurons at $r$. The section in (B) shows dA4-derived axonal trajectories ascending from the caudal hindbrain toward the developing cerebellum. The section in (C) shows $\mathrm{dA4-derived} \mathrm{axonal} \mathrm{trajectories} \mathrm{that} \mathrm{terminate} \mathrm{in} \mathrm{the} \mathrm{cerebellum.} \mathrm{An} \mathrm{outer} \mathrm{granular} \mathrm{layer} \mathrm{of} \mathrm{the} \mathrm{cerebellum} \mathrm{is} \mathrm{marked} \mathrm{by} \mathrm{Axonin} 1$. Bars, $50 \mu \mathrm{m}$. ION, inferior olivary nuclei; Ax-1, axonin 1. 
exclusively at $\mathrm{r} 7$ and demonstrated that their axonal crossing and growth toward the cerebellum (Figures $4 \mathrm{~B}, \mathrm{C}$ ) is initiated much before their neuronal soma migrate to the ION and establish the mature olivocerebellar circuit (Meredith et al., 2009; Kohl et al., 2015).

\section{Class B: dB1 Interneurons Specification and Fate}

The $\mathrm{dB} 1$ subpopulation is the dorsal most group amongst class $\mathrm{B}$ dINs, ventrally flanking dA4 (Figure 1A). This inhibitory group expresses a combination of molecular markers including Ptfla and Ascl1 in their progenitorial stage, followed by upregulation of Lbx1, Lhx1, Lhx5, and Pax2 (Figures 1B,C) (Gross et al., 2002; Müller et al., 2002; Glasgow et al., 2005; Sieber et al., 2007; Fujiyama et al., 2009; Storm et al., 2009; Hoshino, 2012; Kohl et al., 2015; Nothwang, 2016). Multiple lineages tracing studies in mice and chicks, together with the generation of Ptfla ${ }^{-/-}$mice, have indicated that $\mathrm{dB} 1$ neurons migrate to various locations in the hindbrain and contribute inhibitory outputs to multiple nuclei centers, according to their rhombomeric origin (Sieber et al., 2007; Tashiro et al., 2007; Hori and Hoshino, 2012; Iskusnykh et al., 2016). For instance, dB1/Ptf1a ${ }^{+}$neurons from r2-r5 were found to contribute to the auditory system by settling in the DCN, or the avian homolog nucleus angularis (NA) (Farago et al., 2006; Fujiyama et al., 2009; Kohl et al., 2015; Lipovsek and Wingate, 2018). The DCN, which receives inputs from the auditory nerve as well as from various brain sources, has a complex layered organization that resembles the cerebellum (Soares et al., 2002; Sawtell and Bell, 2013; Trussell and Oertel, 2018). Within the DCN, dB1 $\left(\mathrm{Ptfla}^{+} / \mathrm{lbx}^{+}\right)$derivatives were shown to give rise to multiple GABAergic neuronal cell types such as the inhibitory stellate cells, cartwheel cells, and Golgi cells, as well as to a small glycinergic population within the VCN (Farago et al., 2006; Fujiyama et al., 2009; Schinzel et al., 2021). Moreover, comparative analyses of the origin of different vestibular nuclei in mice and chick embryos have indicated that $\mathrm{dB} 1 / \mathrm{Ptfla}^{+}$neurons from $\mathrm{r} 2-\mathrm{r} 8$ also contribute to the medial, lateral, and descending vestibular nuclei (MVN/LVN/DVN), three relay sensory hubs located in the medial column of the medulla and function to control eye, head and neck movements to maintain balance (Marín and Puelles, 1995; Díaz et al., 2003; Maklad and Fritzsch, 2003; Pasqualetti et al., 2007; Yamada et al., 2007; Straka et al., 2014; Kohl et al., 2015; Lipovsek and Wingate, 2018; Díaz and Puelles, 2019; Lunde et al., 2019), as well as to the spinal trigeminal nucleus and solitary nucleus (Yamada et al., 2007). This wealth of derivatives raises the question as to how distinct lineages of $\mathrm{dB} 1$ inhibitory neurons are being generated from the longitudinal $\mathrm{Ptfla}^{+}$progenitorial domain in the hindbrain RL.

\section{Axonal Projection Patterns}

Multiple axonal labeling studies and genetic fate maps uncovered the projections of the auditory and vestibular centers, which are likely to contain $\mathrm{dB} 1 / \mathrm{Ptfla}^{+}$neuronal derivatives. For instance, the mammalian DCN/avian NA was found to form local medullary connections with the ipsilateral mammalian VCN/avian NM. This center projects to the nearby SON and the lateral lemniscal nuclei and extends projections through the ipsi and contralateral DF into the mammalian inferior colliculi/avian nucleus mesencephalicus lateralis pars dorsalis (MLD) in the anterior midbrain, an auditory center that transmits inputs to the medial geniculate body of the thalamus (Figure 5B) (Rubel and Parks, 1975; Takahashi and Konishi, 1988; Puelles et al., 1994; Cant and Benson, 2008; Krützfeldt et al., 2010a,b; Trussell and Oertel, 2018). Moreover, the different vestibular nuclei project via multiple ipsi and contralateral tracts to either descend to the spinal cord via the lateral or medial vestibulospinal tract or ascend via the medial longitudinal fascicle (MLF) to the midbrain Edinger-Westphal nuclei (EW, an autonomic parasympathetic component of the oculomotor nuclear complex that connects to the orbit ciliary ganglion) (Figure 5C). Other vestibular neurons form local connections between different vestibular nuclei or ascend to the cerebellum via vestibulocerebellar mossy fibers (Figure 5C) (Akert et al., 1980; Díaz et al., 1998; Straka et al., 2001, 2014; Balaban, 2003; Barmack, 2003; Pasqualetti et al., 2007; GottesmanDavis and Peusner, 2010; Chagnaud et al., 2017; Di Bonito et al., 2017; Lunde et al., 2019; Ando et al., 2020). Albeit the importance of these brainstem circuits, the association of these multiple axonal projections to the $\mathrm{dB} 1$ subgroup was not fully confirmed, as neurons within different vestibular nuclei were found to derive from additional dINs such as dA1 and $\mathrm{dB} 2$ subgroups.

To address this issue, we aimed at tracing the axons of $\mathrm{dB} 1$ dINs, from soon after their birth up to their arrival at their target sites. By electroporating Ptfla-enhancer element upstream to Cre recombinase along with conditional GFP reporter plasmids we were able to reliably label $\mathrm{dB} 1\left(\mathrm{Ptf} 1 \mathrm{~A}^{+} / \mathrm{Lhx} 1 / 5^{+} / \mathrm{Pax} 2+\right)$ dINs and demonstrate their multiple axonal projections that extended at subsequent time points (Figure 5A) (Meredith et al., 2009; Kohl et al., 2015). The first-appearing axons crossed the floor-plate and turned rostrally, joining either the contralateral MLF or the contralateral DF. Next, an ascending ipsilateral axonal tract began to project along with the ipsilateral MLF. Finally, two more ascending ipsilateral projections were evident; one emerged from a medial position forming an ipsilateral funiculus (LF) whereas the other elongated in a dorsal position forming an ipsi dorsal funiculus (DF) (Figure 5A). These axons projected and terminated in the medulla, cerebellum, MLD, and EW nuclei, invariably with the above-mentioned axonal routes of the vestibular and auditory nuclei (Figures 5B,C). These findings enabled us to connect the $\mathrm{dB} 1$ lineage with typical hindbrain tracts and target sites, as well as to uncover that $\mathrm{dB} 1$ axons begin to project toward different targets before their cell body migrate and settle in their final VN/CN centers (Kohl et al., 2015).

Taken together, the inhibitory $\mathrm{dB} 1 / \mathrm{Ptfla}^{+}$subclass contributes to various types of brainstem nuclei, projects into discrete tracts at different time points, and synapses at multiple target sites. Their various fates and functions indicate that the $\mathrm{dB} 1$ neuronal group is likely to be composed of several subpopulations, each with its birth-time and fate. Intriguingly, since a single rhombomere gives rise to different $\mathrm{dB} 1$ axonal tracts and to several neuronal lineages that settle at various nuclei centers, it 

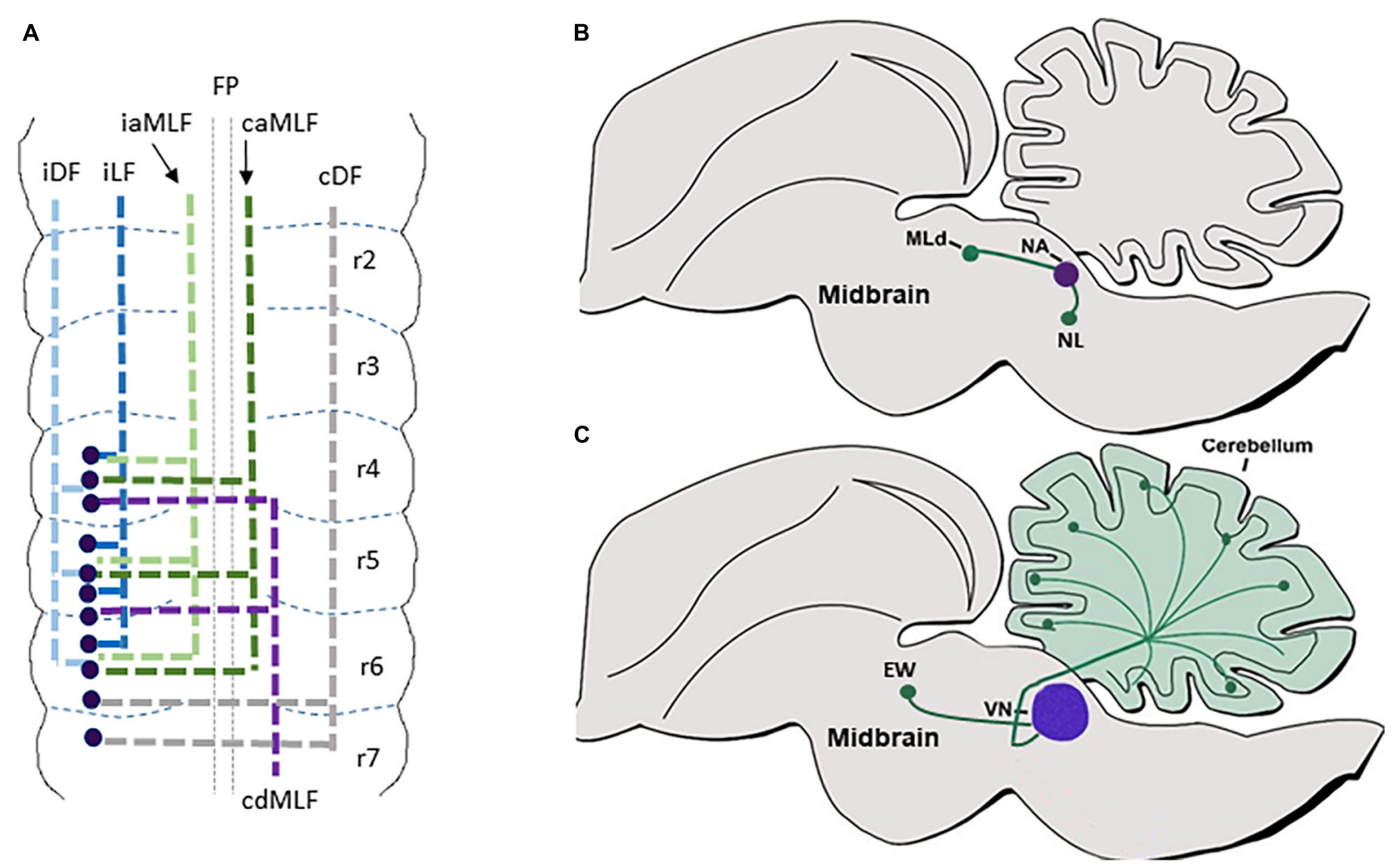

FIGURE 5 | dB1 axonal projections. (A) A summary of dB1 axonal projections as seen in a flat-mount view of E6.5 chick embryonic hindbrain. $\mathrm{dB} 1 \mathrm{cell}$ bodies are shown as blue dots. Each axonal tract is shown in a different color and refers to a distinct funiculus. (B) A schematic sagittal section of the main auditory axonal circuits of dB1 subclass in the chick hindbrain. dAB1-derived NA (purple circle) is shown to project axons (green lines) to the NL in the medulla or to the MLd in the midbrain (green circles). (C) A schematic sagittal section of the main vestibular axonal circuits of dB1 subclass in the chick hindbrain. dA1-derived VN (purple circle) is shown to project axons (green lines) to the cerebellum and EW nuclei in the midbrain (green circles). FP, floor plate; iDF, ipsi dorsal funiculus; iLF, ipsilateral funiculus; iaMLF; ipsilateral ascending; caMLF, contralateral ascending medial longitudinal funiculus; CdMLF, contralateral descending medial longitude in nucleus laminaris; MLD, mesencephalicus lateralis pars dorsalis; VN, vestibular nuclei; EW, Edinger-Westphal.

is likely that several rhombomere-specific regulators, which are unknown yet, act in defined spatiotemporal patterns to provide such intra-segmental diversity.

\section{Class B: dB2 Interneurons Specification and Fate}

The $\mathrm{dB} 2$ subpopulation is an excitatory group that develops in $\mathrm{r} 2-\mathrm{r} 6$, ventral to $\mathrm{dB1}$ (Figures $\mathbf{1 A , B}$ ). In contrast to its neighboring subgroups, it does not express Ascl1 in its progenitorial state but expresses Lbx1 and Phox $2 \mathrm{~b}$ upon differentiation (Figure 1B). In r4-r6, dB2 INs also express Phox2a, indicating at least two dB2 subgroups in the hindbrain (Sieber et al., 2007; Dubreuil et al., 2009; Rose et al., 2009a,b; Storm et al., 2009). Interestingly, this subpopulation is hindbrainspecific as no equivalent spinal dIN subgroup exists. Genetic fate map studies in rodents, combined with the generation of Lbx1/Phox 2b-deficient mice, uncovered the contribution of dB2 $\left(\mathrm{Lbx} 1^{+} / \mathrm{Phox} 2 \mathrm{~b}^{+}\right)$neurons to the RTN/parafacial respiratory group (pFRG) in the ventral medulla. This glutamatergic center relays modulatory input to the preBötC to control respiration rhythm by chemosensing $\mathrm{CO}_{2}$ levels in the blood
(Stornetta et al., 2006; Pagliardini et al., 2008; Dubreuil et al., 2009; Guyenet et al., 2009; Thoby-Brisson et al., 2009; Feldman and Kam, 2015; Ikeda et al., 2015, 2017). Notably, the dB2RTN precursors begin to express Atoh1 once they mature, serving as the only non-dA1 subgroup which requires this gene for its development (Dubreuil et al., 2009; Rose et al., 2009a,b; van der Heijden and Zoghbi, 2018). Elegant lineage tracing strategies uncovered that $\mathrm{dB} 2 / \mathrm{Phox}_{2} \mathrm{~b}^{+}$RTN neurons arise from $\mathrm{r} 3 / \mathrm{r} 5 / \mathrm{Krox} 20^{+}$domains, and as such, mouse or human mutations in Phox $2 \mathrm{~b}$ or Krox20 lead to respiratory rhythm impairments (Jacquin et al., 1996; Weese-Mayer et al., 2005; Dubreuil et al., 2008; Pagliardini et al., 2008; Champagnat et al., 2009; Thoby-Brisson et al., 2009). In addition, dB2 neurons are also likely to contribute to the VN complex; fate map analysis of Hoxb1 ${ }^{\mathrm{GFP}}$ reporter mice showed that the LVN, which regulates the vestibulospinal reflex to maintain proper balance via the back and limb muscles, originate from Hoxb1 ${ }^{+}$precursor neurons in $\mathrm{r} 4$ which co-express the $\mathrm{dB} 2$ markers Lbx $1 /$ Phox $2 \mathrm{~b} / \mathrm{Phox} 2 \mathrm{a}$, and fails to form in Hoxb1-null mice (Díaz et al., 1998; Maklad and Fritzsch, 2003; Chen et al., 2012; Di Bonito et al., 2015). Yet, although detailed chick/mouse fate map studies have previously delineated the rhombomeric origin of all $\mathrm{VN}$ subtypes in the 
hindbrain, (Díaz et al., 1998; Cambronero and Puelles, 2000; Pasqualetti et al., 2007), their association to dB2 dINS that originate from various rhombomeres has not been shown yet.

\section{Axonal Projection Patterns}

As described above, intersectional fate maps demonstrated the contribution of $\mathrm{dB} 2 \mathrm{dINs}$ to respiratory and vestibular circuits. However, knowledge regarding the axonal patterns of genetically-identified dB2 dINs, either before their arrival to their nuclei centers or after they settle in their final destinations, is sketchy. Data from Atoh $1^{\text {lacZ }}$ reporter mice have demonstrated that mature pFRG/RTN neurons extend LacZ-labeled axons toward the ipsilateral preBötC (Figure 6) (Huang et al., 2012). Furthermore, multiple anterograde/retrograde studies, as well as electrophysiological analyses in the mature brainstem, have demonstrated that the pFRG/RTN relays glutamatergic inputs to other hindbrain areas, in addition to the pre-BötzC centers, such as to the ventral respiratory column in the medulla, the ipsilateral, ventrolateral, and intermediate subnuclei of the NTS, the $\mathrm{PBN} / \mathrm{KF}$ at the dorsolateral pons, and the noradrenergic A5 cluster (Stornetta et al., 2006; Dubreuil et al., 2009; Guyenet et al., 2009; Thoby-Brisson et al., 2009; Bochorishvili et al., 2012; Feldman and Kam, 2015; Ikeda et al., 2017). Despite these findings, it is not fully clear whether all these projections arise from $\mathrm{dB} 2$-derived neurons in the pFRG/RTN or from other types of neurons that cluster in these heterogenic nuclei.

In addition, r4-derived VLN neurons, which are likely to originate from $\mathrm{dB} 2$ subpopulation, were found to project to the ipsilateral vestibulospinal tract (LVST), that descends along the spinal cord to connect to motor neurons of the extensor musculature of the limbs and the trunk (Figure 6). These LVST axons extend in a ventromedial direction toward the inferior olive, thereupon turning and descending through the medullary reticular formation within the ipsilateral ventral funiculus, and terminates at cervical and lumbosacral levels on INs residing in the ventral gray column (Díaz et al., 1998; Auclair et al., 1999; Straka et al., 2001, 2014; Maklad and Fritzsch, 2003;
Pasqualetti et al., 2007; Chen et al., 2012; Liang et al., 2014; Di Bonito et al., 2015; Lunde et al., 2019).

Altogether, the complex reality of several dB2 subgroups as recognized by their unique transcriptional profile and fates among different rhombomeres, require further fate map investigations to reveal their precise destination and axonal connection to different somatosensory brain circuits.

\section{Class B: dB3 Interneurons Specification and Fate}

The excitatory $\mathrm{dB} 3$ neuronal population is born ventrally to the $\mathrm{dB} 2$ subgroup in $\mathrm{r} 2-\mathrm{r} 6$, and to $\mathrm{dB} 1$ in $\mathrm{r} 7$ (Figure 1A). $\mathrm{dB} 3$ is derived of a Ascl1 ${ }^{+}$progenitor domain and has a unique transcriptional profile of Lbx1/Tlx3/Lmx1b/Pou4f1/FoxP2 (Cheng et al., 2004; Mizuguchi et al., 2006; Sieber et al., 2007; Pagliardini et al., 2008; Storm et al., 2009; D’Autréaux et al., 2011; Gray, 2013) (Figures 1B,C). Multiple fate map studies of $\mathrm{Ascl}^{+} / \mathrm{Lbx}^{+} / \mathrm{Tlx}^{+}$neurons, as well as knockout of these genes, have suggested that $\mathrm{Ascl}^{+} / \mathrm{Lbx}^{+} / \mathrm{Tlx}^{+}$neurons contribute to the development of somatic sensory nuclei in the hindbrain, such as the spinal trigeminal nuclei (Sp5) and the principal trigeminal nucleus $(\operatorname{Pr} 5)$, that relay various sensory modalities including temperature, touch, and pain from the ipsilateral faces (Qian et al., 2001, 2002; Sieber et al., 2007; Kim et al., 2008). Yet, genetic intersection approaches to target only dB3 dINs, rather than various $\mathrm{Ascl} 1^{+} / \mathrm{Tlx} 3^{+} / \mathrm{Lbx}^{+}{ }^{+}$-expressing neurons, have not been performed. Hence, the involvement of this subgroup in the brainstem somatic sensory system as well as its possible contribution to other hindbrain nuclei centers needs to be fully determined.

\section{Axonal Projection Patterns}

Axonal projections of brainstem relay somatic sensory neurons have been well described in adult brains. For instance, neurons originating from different subparts of the Sp5 nucleus project to several thalamic domains via the contralateral ventral trigeminal tract, such as the ventral posteromedial nucleus, the posterior group, and the region intercalated between the anterior pretectal

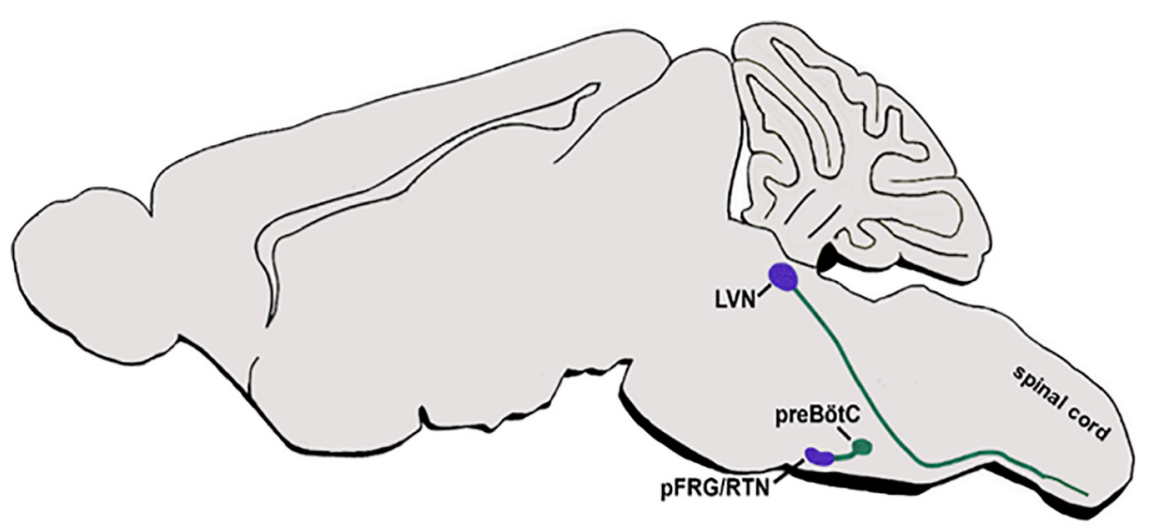

FIGURE 6 | dB2 axonal projections. A schematic sagittal section of the main respiratory and vestibular circuits of dB2 subclass. dB2-derived pFRG or LVN nuclei (purple circles) are shown to project axons (green lines) to the hindbrain preBötC nuclei or to descend toward the spinal cord motor neurons (green circles). pFRG/RTN, parafacial respiratory group/reticulotegmental nucleus; LVN-lateral vestibular nucleus; preBötC-pre Bötcinger. 
and the medial geniculate nuclei. Sp5 neurons were also shown to project to the contralateral inferior colliculus in the midbrain, as well as to the local Pr5 nuclei and the granular, bushy, and stellate cell layers of the brainstem CN (Veinante et al., 2000; Zhou and Shore, 2006; Zeng et al., 2011; Heeringa et al., 2018). Furthermore, different subsets of $\operatorname{Pr} 5$ neurons were shown to project to centers in the medulla and pons such as the ipsi and contralateral solitary tract, the rostroventrolateral reticular nucleus, the $\mathrm{AMB}$ nucleus, the lateral reticular nucleus, and the ipsilateral PBN, as well as to the red nucleus (de Sousa Buck et al., 2001; Pinto et al., 2007). Interestingly, Lbx1 ${ }^{-/-}$ mice were reported to extend misrouted tracts, where medullary longitudinal fibers shifted from ventral to more dorsal positions (Pagliardini et al., 2008). Yet, whether these fibers extended from $\mathrm{dB} 3 / \mathrm{Lbx}^{+}$neurons in the Sp5/Pr5 nuclei, is not fully clear. Moreover, as Sp5 and Pr5 nuclei are divided into several subcenters, the relative contribution of $\mathrm{dB} 3$ neurons to these nuclei and their multiple axonal projections has to be deciphered.

\section{Class B, dB4 Interneurons Specification and Fate}

The dB4 subpopulation is the ventral most group of hindbrain dINs, located ventral to $\mathrm{dB} 3$ (Figure 1A). Derived from Neurog1/2 progenitor domain, dB4 INs are inhibitory and express a combination of markers including Lhx1/5, Pax2, bHLHb5, Wilms tumor protein (Wt1), and presumably also the double sex/male abnormal 3 (DMRT3) (Figures 1B,C) (Gross et al., 2002; Müller et al., 2002; Sieber et al., 2007; Gray, 2008; Pagliardini et al., 2008; Hernandez-Miranda et al., 2017; Schnerwitzki et al., 2020). Notably, their spinal cord homolog group dI6, which shares a similar molecular profile and DV localization as $\mathrm{dB} 4$, was found to consist of three distinct subgroups, based on their singular or co-expression of Wt1 and/or DMRT3 (Gross et al., 2002; Helms and Johnson, 2003; Vallstedt and Kullander, 2013; Hernandez-Miranda et al., 2017;
Schnerwitzki et al., 2020). dI6 neurons were found to migrate to the ventral horn of the spinal cord and to coordinate locomotion in different mammals (Andersson et al., 2012; Vallstedt and Kullander, 2013; Haque et al., 2018; Schnerwitzki et al., 2018). Nevertheless, it is currently uncertain whether the dB4 subgroup is also heterogeneous in Wt1/DMRT3 expression and whether it also plays a role in locomotion coordination.

In a previous study, hindbrain $\mathrm{WT}^{+}$neurons were discovered in the AP nucleus, suggesting that $\mathrm{dB} 4 / \mathrm{WT}^{+}$neurons may be fated to contribute an inhibitory module to this nucleus, in addition to the presence of excitatory dA3 neurons in the AP (Sharma et al., 1992). Recent data have uncovered an additional fate of $\mathrm{Wt1}^{+}$neurons in the caudal ventral respiratory group (cVRG) (Schnerwitzki et al., 2020). This neuronal cluster is positioned in the caudal-most part of the respiratory column and is known to participate in the activation of motor neurons in the cervical spinal cord, which in turn innervate the diaphragm muscles, leading to their contraction and thereby to inspiration (Ezure et al., 2003; Alheid and McCrimmon, 2008). As such, ablation of $\mathrm{Wt}^{+}$neurons resulted in the death of neonates due to the inability to initiate respiration, suggesting a vital role for $\mathrm{Wt1}^{+}$neurons in breathing (Schnerwitzki et al., 2020). Since WT expression in the dINs is restricted to the dB4 subgroup, these results strongly suggest the contribution of the $\mathrm{dB} 4$ subgroup to respiratory control. Yet, as $\mathrm{dB} 4 \mathrm{dINs}$ appear along the entire hindbrain AP axis, this subgroup is likely to contribute to additional brainstem nuclei.

\section{Axonal Projection Patterns}

In the spinal cord, dI6/DMRT ${ }^{+}$neurons project ipsi and contralateral axons that innervate somatic motoneurons of tibialis anterior and/or gastrocnemius (Andersson et al., 2012; Vallstedt and Kullander, 2013). Although dB4/WT ${ }^{+}$ subpopulation was found to contribute to the AP and cVRG (Figure 7) (Sharma et al., 1992; Schnerwitzki et al., 2020), genetic labeling of their axons and target sites has not been performed

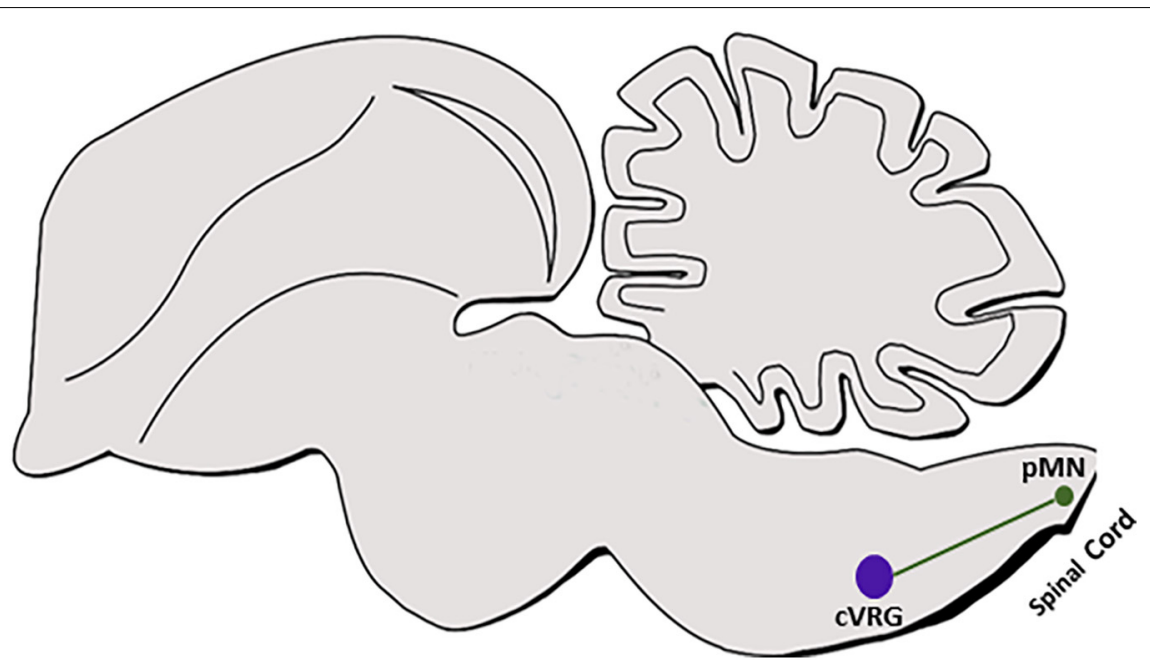

FIGURE 7 | dB4 axonal projections. A schematic sagittal section of dB4/WT1+ axonal circuit. dB4-derived cVRG (purple circle) is shown to project axons (green line) to pMNs in the cervical spinal cord (green circle). WT, Wilm's tumor 1; cVRG, caudal ventral respiratory group; pMN, pre motor neurons. 
as of yet. Hence, while multiple ipsi and contralateral axonal projections are known to arise from the AP to target multiple nuclei sites in the medulla and the upper brain (van der Kooy and Koda, 1983; Shapiro and Miselis, 1985; Price et al., 2008; Stein and Loewy, 2010), it is not yet clear whether any of these targets are innervated by $\mathrm{dB} 4$ axons. In addition, cVRG sends commissural axons that descend in the ventromedial medulla toward their premotor neuronal targets in the contralateral cervical spinal cord that are responsible to activate inspiratory and expiratory motor neurons (Ezure et al., 2003; Alheid and McCrimmon, 2008). Although this tract is likely to be projected from the $\mathrm{dB} 4 / \mathrm{WT}^{+}{ }^{\mathrm{cVRG}}$ neurons (Figure 7) (Schnerwitzki et al., 2020), tracing of genetically labeled dB4 axons is required to fully support this data as well as to identify additional projections from more rostral dB4 neurons.

\section{MOLECULAR REGULATORS THAT CONTROL AXONAL GROWTH OF HINDBRAIN dINS}

Axonal growth is a multi-event process that includes axonogenesis, pathfinding, arborization, and establishment of terminals on appropriate postsynaptic structures (Chédotal and Richards, 2010; Stoeckli, 2018; Comer et al., 2019). As different $\mathrm{dA} / \mathrm{dB}$ subgroups display multiple types of axonal routes, they are likely to share common molecular cues that, for instance, guide their axonal crossing across the FP or extend their projections in fasciculated funiculi. In parallel, each neuronal subtype is also likely to respond to individual cues that determine its particular axonal pattern. Identification of such cues is crucial for uncovering how brainstem circuitries are assembled during normal development or misassembled in neurodevelopmental disorders. Less knowledge exists on $\mathrm{dA} / \mathrm{dB}$ hindbrain axons, as opposed to their $\mathrm{dI} / \mathrm{dB}$ spinal cord homologs. A summary of the knowledge on $\mathrm{dA} / \mathrm{dB}$ axonal growth regulation is presented here, according to different types of molecules. Notably, although dINs undergo extensive neuronal cell body migration in parallel to growing axons, this topic is not discussed here.

\section{Transcription Factors \\ Lhx}

Lim-HD proteins control multiple aspects of neuronal development, including axonal guidance (Hobert and Westphal, 2000; Kania et al., 2000; Shirasaki and Pfaff, 2003; Wilson et al., 2008; Avraham et al., 2009; Roy et al., 2012). In particular, different Lhx protein members are expressed in the spinal cord and forebrain and regulate axonal growth and neurotransmitter type of different neuronal populations (Pillai et al., 2007; Wilson et al., 2008; Avraham et al., 2009; Palmesino et al., 2010; Chou and Tole, 2019). In the hindbrain, particular $\mathrm{dA} / \mathrm{dB}$ subgroups express different Lhx's proteins; Lhx2/9 are specific to dA1, while Lhx1/5 are specific to dB1 and dB4 (Gray, 2008; HernandezMiranda et al., 2017). To address whether these factors govern the specific axonal paths of each subgroup, we have switched between the expression of the dA1-specific Lhx2/9 and the dB1-specific Lhx1/5 genes, such that each neuronal population was forced to express the Lhx's of the other. This modification did not affect their specification but was sufficient to misdirect axonal patterns and target sites of one subgroup to phenocopy the patterns of the other (Kohl et al., 2012, 2015). As such, dA1 axons shifted to target the Purkinje cell layer, and dB1 axons terminated at the granular cell layer of the cerebellum, demonstrating the important regulatory role of the Lhx code in assembling $\mathrm{dA} / \mathrm{dB}$ axonal circuits in the developing brainstem. To uncover the mechanism by which Lhx proteins control $\mathrm{dA} / \mathrm{dB}$ axonal patterns, downstream effector genes that act as guidance cues should be uncovered. Notwithstanding is the fact that although Lhx2/9 or Lhx1/5 are longitudinally expressed in $\mathrm{dA} 1 / \mathrm{dB} 1$ subgroups along with the entire hindbrain, several axonal trajectories extend from each subgroup (Figures 2, 5), indicating that other factors guide the growth of axons within each group, in addition to Lhx proteins.

\section{Hox}

As Hox genes are fundamental for the segmental identity and patterning of rhombomeres, their involvement in regulating axonal projections of dINs that originate from individual rhombomeres has been suggested. Well-designed fate map analyses of individual rhombomeres using Hox-specific enhancers, together with the characterization of mutant mice/zebrafish, uncovered the role of Hox genes in governing vestibular, trigeminal, branchial, auditory, pre-cerebellar, and somatosensory nuclei projections (Carpenter et al., 1993; Marín and Puelles, 1995; Gavalas et al., 1997; Glover, 2000; del Toro et al., 2001; McClintock et al., 2002; Maklad and Fritzsch, 2003; Farago et al., 2006; Oury et al., 2006; Pasqualetti et al., 2007; Geisen et al., 2008; Narita and Rijli, 2009; Di Bonito et al., 2013, 2015; Lipovsek and Wingate, 2018; Beiriger et al., 2021). For example, Hoxa 2 was found to be required for the contralateral projections of dA1-derived AVCN axons to the medial nucleus of the trapezoid body (MNTB), which aberrantly innervated the ipsilateral MNTB in Hoxa2 mutants (Di Bonito et al., 2013). Hoxa2 was also found to be involved in the topographic specificity of axons extending from the dB3-derived Pr5 nuclei to the thalamic ventral posterior medial (MPV) nucleus (Oury et al., 2006). Yet, many of these studies focused more on neuronal cell body migration rather than on axonal projections or did not associate defined projection patterns with genetically identified $\mathrm{dA} / \mathrm{dB}$ subclasses. Hence, more data is required to further illuminate the role and manner of action of Hox genes in governing axonal growth decisions of particular $\mathrm{dA} / \mathrm{dB}$ dorsal INs.

\section{Zic}

The transcription factor Zic1, a member of the Zinc Finger of the Cerebellum (Zic) family, is expressed in PGN neurons in the ventral hindbrain. Zic1 was found to drive axon laterality choice to the ipsilateral, rather than the contralateral pontocerebellar tract, by inhibiting axonal midline crossing (DiPietrantonio and Dymecki, 2009). PGN neurons belong to dA1/Atho ${ }^{+}$MF neuronal subgroup that arises in the caudal hindbrain. While Zicl effectors that inhibit dA1 axonal crossing are not known, previous studies in the spinal cord or the upper brain have found 
that Eph receptors are induced by Zic proteins to activate the repulsive Eph-ephrin signaling cue (García-Frigola et al., 2008; Lee et al., 2008; Escalante et al., 2013). Future studies are required to reveal whether Zic1 upregulates Eph receptors in MF neurons and whether it impacts axonal growth decisions in additional neuronal subgroups along the rhombic lip that extend axons to both sides of the hindbrain.

\section{Axonal Guidance Cues Robo/Slit}

Roundabout (Robo) family of transmembrane receptors and their soluble ligands Slit are chemorepulsive cues fundamental for commissural axonal guidance in the CNS (Tessier-Lavigne and Goodman, 1996; Dickson and Gilestro, 2006). Mutations in human Robo genes were found to disrupt hindbrain axon crossing in patients with horizontal gaze palsy with progressive scoliosis (Jen et al., 2004). Multiple studies in mice have shown a conserved role of Robo/Slit signaling in guiding commissural axons in the hindbrain, before and after crossing the FP (Lee et al., 2001; Causeret et al., 2002; Bloch-Gallego et al., 2005; Howell et al., 2007; Tamada et al., 2008; Bouvier et al., 2010; Renier et al., 2010; Mirza et al., 2013; Friocourt et al., 2019). For instance, PCN/MF neurons and auditory AVCN neurons, that are both generated from the $\mathrm{dA} 1 / \mathrm{Atoh}^{+}$group, require Robo/Slit signaling for axonal elongation from the dorsal rhombic lip toward the FP and for crossing the midline, which fails to occur in Robo3 mutant mice (Marillat et al., 2004; Renier et al., 2010). Similarly, ION/CF neurons, that originate from $\mathrm{dA} 4 / \mathrm{Ptfla}^{+}$progenitors and extend contralateral fibers toward the cerebellum, require Robo3 signaling for their axonal crossing, as shown by their abnormal extension of ipsilateral processes in Robo3 deficient mice (Marillat et al., 2004). At variance, the knockout of other Robo/Slit members such as Robo1/2 or Slit1/2 did not impair the midline crossing of PCN axons, although it severely affected their cell body migration (Geisen et al., 2008). Interestingly, Robo1/3 expression was found to be induced by Lhx2/9 and to control axonal growth of thalamocortical and spinal neurons (Wilson et al., 2008; Chatterjee et al., 2012). These findings may suggest that the regulatory role of $\mathrm{Lhx} 2 / 9$ on dA1 axons (Kohl et al., 2012, 2015) is mediated by Robo3. Yet, an opposite role of $\operatorname{Lhx} 2 / 9$ to prevent the expression of Slit was recently found in retinal ganglion axons (Yang et al., 2020), indicating that the inductive or inhibitory role of Lhx2/9 on Robo/Slit signaling is context-dependent. Another interesting upstream regulator of Robo3 in the hindbrain is Hoxa2; In the dA1-derived AVCN, Robo3 expression was found to be induced by Hoxa 2 and to be necessary to guide AVCN axons to project into the contralateral medulla (Di Bonito et al., 2013).

\section{Adhesion Molecules}

Cell adhesion molecules (CAMs) are instructive for axonal pathfinding, elongation, and fasciculation (Walsh and Doherty, 1997; Colman and Filbin, 1999; Hirano et al., 2003; BlochGallego et al., 2005; Pollerberg et al., 2013). Several previous studies discovered various cadherin (Cdh) subtypes (i.e., N-Cdh, E-Cdh, Cdh6/8/11, and protocadherins7/10) that are expressed in $\mathrm{dA} / \mathrm{dB}$-derived nuclei, such as in the trigeminal, raphe, inferior olive, pre-cerebellar, and vestibular nuclei (Shimamura et al., 1992; Redies and Takeichi, 1993; Korematsu and Redies, 1997; Taniguchi et al., 2006; Neudert and Redies, 2008). Confirmation of their role in axonal guidance was shown in the LRN, ECN, and $\mathrm{PN}$, which originate from $\mathrm{dA} 1 \mathrm{dIN}$ and require $\mathrm{N}-\mathrm{Cdh}$ and Cdh11 for their soma and axonal migration toward the FP, or Cdh7 for their projection toward the cerebellum (Taniguchi et al., 2006; Kuwako et al., 2014). Interestingly, an interplay between Robo and N-Cdh was shown to guide spinal commissural axons of $\mathrm{dI} 1 / \mathrm{dI} 2$ subgroups into longitudinal tracts that participate in the spinocerebellar projection (Sakai et al., 2012), raising the possibility that these cues also cooperate in hindbrain PCN/ION axonal migration. Two additional adhesion molecules, the NgCAM-related CAM Nr-CAM and its receptor TAG-1 were also found to be expressed in the caudal hindbrain; Nr-CAM is expressed in the dA4-derived ION neurons whereas Tag1 is restricted to the dA1-derived PCN (Backer et al., 2002). As Tag1 and $\mathrm{Nr}$-CAM play crucial roles in commissural axon guidance across the spinal cord midline (Stoeckli and Landmesser, 1995; Lustig et al., 2001), it remains to be determined whether they play a similar role in hindbrain axons. Finally, nectin-like proteins $(\mathrm{Necl} 1 / 3)$ were found to be involved in the regulation of commissural axonal trajectories in the anterior hindbrain, as their perturbation caused abnormal fasciculation in the form of failure to turn longitudinally at the contralateral side of the rat hindbrain (Okabe et al., 2004). Although not genetically identified, their position suggests they are likely to arise from the $\mathrm{dA} 1$ subgroup in $\mathrm{r} 1$.

\section{Eph-Ephrin}

Eph proteins, including the Eph receptors and their ligands ephrins, are fundamental for axonal guidance in the CNS (Cheng et al., 1995; Cramer and Gabriele, 2014; Klein and Kania, 2014; Milinkeviciute and Cramer, 2020). A series of elegant studies in the auditory hindbrain uncovered the role of EphA4/B2/B3 receptors in regulating axonal connectivity of the dA1-derived NM-NL binaural circuit in avian, or in the projection of the VCN nuclei, which consist of $\mathrm{dA} 1$ and $\mathrm{dB} 1$ neurons, to the contralateral MNTB in mice. Disruption in these receptors in chick or mouse embryos resulted in axonal misrouting and various targeting errors (Cramer et al., 2004, 2006; Huffman and Cramer, 2007; Hsieh et al., 2010; Abdul-latif et al., 2015). EphAephrinA interactions also play a role in guiding the turning point of axons that project from caudal vestibular neurons (cC-VC) once they cross the midline and extend longitudinally toward the cerebellum (Zhu et al., 2006). Although the genetic identity of these neurons was not shown in this study, their cell body position and axonal patterns suggest that they originate from the dA1 subgroup. Finally, EphA-ephrinA interaction was also suggested to guide ION axons that derive from the dA4 subgroup to their correct targets in the cerebellum (Nishida et al., 2002). Interestingly, data from the spinal cord suggested that limb innervation by lateral motor column neurons depends on EphA4, which is a downstream target of Lhx1 (Kania et al., 2000), raising the possibility that also in the hindbrain, Lhx proteins, which were found to control dA1/dB1 axonal projections (Kohl et al., 2012, 2015), are upstream to Eph-ephrin signaling. 


\section{Netrin/Deleted in Colorectal Cancer}

Multiple pieces of evidence have shown the involvement of the chemoattractant molecule Netrin and its receptor Deleted in Colorectal Cancer (DCC) in hindbrain commissural axons (Keino-Masu et al., 1996; Guthrie, 1997; Bloch-Gallego et al., 2005). For instance, dA1-derived VCN axons, which express DCC, cross the midline to target the contralateral superior olivary complex (SOC). Yet, mice mutated for DCC lack VCN axonal outgrowth (Howell et al., 2007). Moreover, dA1-derived PCN, dA4-derived ION, or dB3-derived Pr5 trigeminal nuclei draw their axons to cross the midline and ascend in defined funiculi toward upper brain regions. Their projection toward the midline, crossing of the FP, and post-crossing trajectories was severely impaired in Netrin or DCC mutants (Yee et al., 1999; Alcantara et al., 2000; Causeret et al., 2002; de Diego et al., 2002; Mirza et al., 2013; Shoja-Taheri et al., 2015; Dominici et al., 2017). Moreover, recent studies uncovered that in the aforementioned knockouts, some of these nuclei project abnormal axons into the PNS along the trigeminal, auditory, and vagal nerve routes (Moreno-Bravo et al., 2018; Yung et al., 2018). Together, these data indicate that the netrin-DCC signaling system is required for establishing proper axonal projections of auditory, pre-cerebellar, and somatosensory hindbrain neurons together with maintaining a clear CNS-PNS boundary in this region.

\section{Neuropilin/Semaphorin}

The role of the neuropilin (Npn) receptors and their semaphorin (Sema) ligands in axonal growth is well known in various sensory and motor neural systems (Neufeld et al., 2002; MeléndezHerrera and Varela-Echavarría, 2006; Pasterkamp, 2012). In the hindbrain, Nrp1 and Sema3A were found to form gradients across the projections of A1-derived pontine axons (Solowska et al., 2002), whereas Npn-1/2 were shown to be expressed in axons projecting from the NTS, which contain dA3-derived neurons (Corson et al., 2013). These spatiotemporal expression patterns indicate that the Npn and Sema families of axon guidance molecules are potential molecular regulators for dA1 and dA3 axonal trajectories. Furthermore, previous data from zebrafish embryos have shown that hindbrain axons that project along the MLF, require Sema3D to promote their fasciculation, which was mediated by the upregulation of the adhesion molecule L1 CAM (Wolman et al., 2007). As many sorts of axons, including those projecting from several $\mathrm{dA} / \mathrm{dB}$ neuronal subtypes, ascend or descend along the MLF, Npn-Sema signaling may have a broad role in hindbrain axonal guidance.

\section{The RNA Binding Protein Fragile X Mental Retardation Protein}

The RNA binding protein FMRP is broadly expressed in the CNS where it acts as a reversible repressor of specific mRNA translation (Darnell et al., 2001; Davis and Broadie, 2017). Functional loss of FMRP leads to fragile $\mathrm{X}$ syndrome, a neurodevelopmental disorder with severe cognitive impairment (Hagerman et al., 2017). Multiple studies have supported the role of FMRP in axonal development, as, for example, FMRP knockout results in excessive axonal branches in motor neurons and abnormal projection patterns in the forebrain

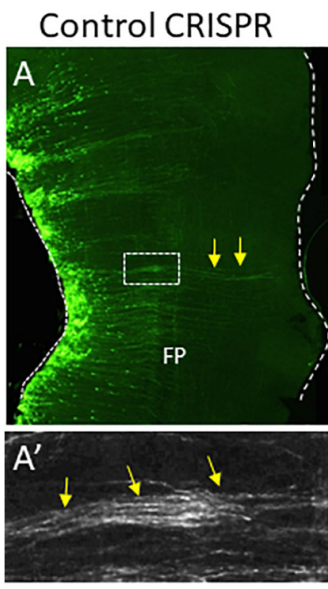

\section{FMRP CRISPR}
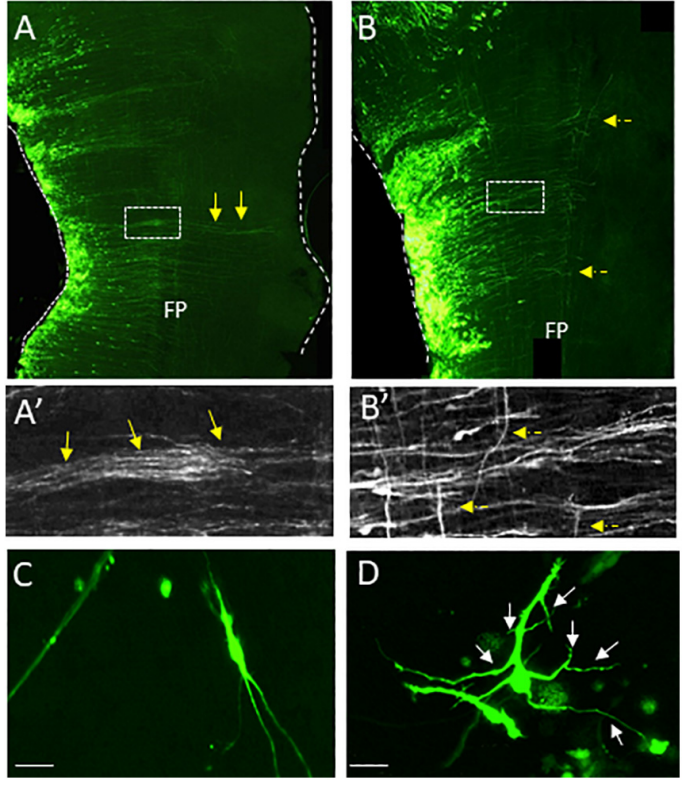

FIGURE 8 | CRISPR-mediated FMRP knockout induces disoriented axonal growth in dA1-derived NM neurons in the chick hindbrain. (A-B') Flat-mounted hindbrains from embryos electroporated with control (A) or FMRP (B) CRISPR/Cas9- guide RNA-GFP plasmids. Electroporated NM axons are GFP-labeled. Higher-magnification views of the boxed areas in $(\mathbf{A}, \mathbf{B})$ are shown in $\left(\mathbf{A}^{\prime}, \mathbf{B}^{\prime}\right)$. Yellow arrows indicate aligned axons that cross the hindbrain midline $\left(\mathbf{A}, \mathbf{A}^{\prime}\right)$. Dashed yellow arrows indicate disoriented axons $\left(\mathbf{B}, \mathbf{B}^{\prime}\right)$. (C,D) Cell cultures from GFP-expressing NM neurons that were electroporated with the above-mentioned plasmids. Control cells $\mathbf{( C )}$ project straight and oriented axons. FMRP-knockout cells (D) project over-branching axons Scale bars: $50 \mu \mathrm{m}$. FP, floor plate; NM, nucleus magnocellularis; CRISP, Crisper/Cas9-based plasmids.

(Bureau et al., 2008; Shamay-Ramot et al., 2015; Scharkowski et al., 2018). Notably, FMRP was found to associate with RNAs that encode axonal guidance molecules, such as netrin and Dscam (Jain and Welshhans, 2016; Kang et al., 2019). We have recently uncovered the role of FMRP in dA1-derived neurons of the auditory NM nuclei in the chick. FMRP was found to localize in axons of dA1/NM neurons, and its downregulation led to perturbed axonal pathfinding, delay in midline crossing, excess branching of neurites, and axonal targeting errors during the period of auditory circuit development (Figure 8) (Wang et al., 2020). This finding provided the first in vivo identification of FMRP activity in developing axons in the hindbrain. Further studies are required to elucidate FMRP-downstream RNA targets in $\mathrm{dA} 1$ axons and to uncover whether fragile $\mathrm{X}$ patients suffer from axonal development deficits in the auditory brainstem.

\section{CONCLUDING REMARKS}

The orderly and conserved development of the segmented hindbrain in vertebrates, together with its fundamental roles to transmit and process sensory-motor orders that arrive from the surroundings, or the spinal cord and higher brain centers, makes 
it a fascinating CNS domain. Yet, how it is set to produce diverse neurons that are programmed to assemble dedicated neuronal circuits that execute a wealth of physiological actions, is only partially clear. The ground plan to develop $8 \mathrm{dINs}$ subclasses along the longitudinal axis of the hindbrain which have to respond to antagonistic roof plate and floor plate DV cues along with rhombomere-specific AP positional information is unique. Many important studies along the years have initially mapped definite DV and AP positions in the hindbrain that drive specific axonal tracts and neuronal clustering which in turn give rise to different brainstem nuclei. With the generation of transgenic and mutant animal models, the genetic code of all individual $\mathrm{dA} / \mathrm{dB}$ subtypes has been decoded, enabling investigation of the developmental program, circuit formation, and fate of dA1-4/dB1-4 neuronal subgroups. Yet, many challenges remain to fully correlate the identity of specific dINs in the early embryo with brainstem nuclei and projections at advanced stages, to identify the mechanism by which each $\mathrm{dA} / \mathrm{dB}$ subgroup, which displays a uniform genetic identity along the AP axis, can eventually give rise to different types of neuronal cell types with distinct axonal connections, target sites, and functions, and to uncover how multiple attractive and repulsive axonal cues are orchestrated to guide the step-by-step assembly of multiple axonal projections that arise from individual and/or neighboring dINs in the developing hindbrain.

\section{REFERENCES}

Abdul-latif, M. L., Salazar, J. A. A., Marshak, S., Dinh, M. L., and Cramer, K. S. (2015). Ephrin-A2 and ephrin-A5 guide contralateral targeting but not topographic mapping of ventral cochlear nucleus axons. Neural Dev. 10:27.

Akert, K., Glicksman, M., Lang, W., Grob, P., and Huber, A. (1980). The EdingerWestphal nucleus in the monkey. A retrograde tracer study. Brain Res. 184, 491-498.

Alcantara, S., Ruiz, M., de Castro, F., Soriano, E., and Sotelo, C. (2000). Netrin 1 acts as an attractive or as a repulsive cue for distinct migrating neurons during the development of the cerebellar system. Development 127, 1359-1372. doi: 10.1242/DEV.127.7.1359

Alheid, G., Jiao, W., and McCrimmon, D. (2011). Caudal nuclei of the rat nucleus of the solitary tract differentially innervate respiratory compartments within the ventrolateral medulla. Neuroscience 190, 207-227. doi: 10.1016/J. NEUROSCIENCE.2011.06.005

Alheid, G., and McCrimmon, D. (2008). The chemical neuroanatomy of breathing. Respir. Physiol. Neurobiol. 164, 3-11. doi: 10.1016/J.RESP.2008.07.014

Altman, J., and Bayer, S. A. (1980). Development of the brain stem in the rat. II. Thymidine-radiographic study of the time of origin of neurons of the upper medulla, excluding the vestibular and auditory nuclei. J. Comp. Neurol. 194, 37-56. doi: 10.1002/CNE.901940103

Altman, J., and Bayer, S. A. (1987a). Development of the precerebellar nuclei in the rat: I. The precerebellar neuroepithelium of the rhombencephalon. J. Comp. Neurol. 257, 477-489. doi: 10.1002/CNE.902570402

Altman, J., and Bayer, S. A. (1987b). Development of the precerebellar nuclei in the rat: II. The intramural olivary migratory stream and the neurogenetic organization of the inferior olive. J. Comp. Neurol. 257, 490-512. doi: 10.1002/ CNE.902570403

Altman, J., and Bayer, S. A. (1987c). Development of the precerebellar nuclei in the rat: III. The posterior precerebellar extramural migratory stream and the lateral reticular and external cuneate nuclei. J. Comp. Neurol. 257, 513-528. doi: 10.1002/CNE.902570404

Altman, J., and Bayer, S. A. (1987d). Development of the precerebellar nuclei in the rat: IV. The anterior precerebellar extramural migratory stream and the nucleus

\section{DATA AVAILABILITY STATEMENT}

The original contributions presented in the study are included in the article/supplementary material, further inquiries can be directed to the corresponding author.

\section{AUTHOR CONTRIBUTIONS}

$\mathrm{DH}, \mathrm{AK}, \mathrm{YW}$, and DS-D participated in writing and revising the manuscript and preparing the figures and contributed to the data presented in the manuscript. All authors contributed to the article and approved the submitted version.

\section{FUNDING}

This work was supported by the United States-Israel Binational Science Foundation (2015087 to YW and DS-D), the Israel Science Foundation (1515/16 to DS-D), and the Office of the Chief Scientist, Ministry of Health (3-0000-33793 to DS-D).

\section{ACKNOWLEDGMENTS}

We thank Avihu Klar for his important contribution to the studies presented in Figures 2, 4, 5, and 8.

reticularis tegmenti pontis and the basal pontine gray. J. Comp. Neurol. 257, 529-552. doi: 10.1002/CNE.902570405

Anderson, D. J., Groves, A., Lo, L., Ma, Q., Rao, M., Shah, N. M., et al. (1997). Cell lineage determination and the control of neuronal identity in the neural crest. Cold Spring Harbor Symp. Quant. Biol. 62, 493-504.

Andersson, L. S., Larhammar, M., Memic, F., Wootz, H., Schwochow, D., Rubin, C.-J., et al. (2012). Mutations in DMRT3 affect locomotion in horses and spinal circuit function in mice. Nature 488, 642-646. doi: 10.1038/nature11399

Ando, T., Ueda, M., Luo, Y., and Sugihara, I. (2020). Heterogeneous vestibulocerebellar mossy fiber projections revealed by single axon reconstruction in the mouse. J. Comp. Neurol. 528, 1775-1802. doi: 10.1002/CNE.24853

Auclair, F. O., Marchand, R., and Glover, J. C. (1999). Regional patterning of reticulospinal and vestibulospinal neurons in the hindbrain of mouse and rat embryos. J. Comp. Neurol. 411, 288-300.

Avraham, O., Hadas, Y., Vald, L., Zisman, S., Schejter, A., Visel, A., et al. (2009). Transcriptional control of axonal guidance and sorting in dorsal interneurons by the Lim-HD proteins Lhx9 and Lhx1. Neural Dev. 4:21.

Backer, S., Sakurai, T., Grumet, M., Sotelo, C., and Bloch-Gallego, E. (2002). Nr-CAM and TAG-1 are expressed in distinct populations of developing precerebellar and cerebellar neurons. Neuroscience 113, 743-748. doi: 10.1016/ S0306-4522(02)00221-X

Bae, Y., Kani, S., Shimizu, T., Tanabe, K., Nojima, H., Kimura, Y., et al. (2009). Anatomy of zebrafish cerebellum and screen for mutations affecting its development. Dev. Biol. 330, 406-426. doi: 10.1016/J.YDBIO.2009.04.013

Balaban, C. (2003). Vestibular nucleus projections to the Edinger-Westphal and anteromedian nuclei of rabbits. Brain Res. 963, 121-131.

Barmack, N. H. (2003). Central vestibular system: vestibular nuclei and posterior cerebellum. Brain Res. Bull. 60, 511-541.

Beiriger, A., Narayan, S., Singh, N., and Prince, V. (2021). Development and migration of the zebrafish rhombencephalic octavolateral efferent neurons. J. Comp. Neurol. 529, 1293-1307. doi: 10.1002/CNE.25021

Ben-Arie, N., Bellen, H. J., Armstrong, D. L., McCall, A. E., Gordadze, P. R., Guo, Q., et al. (1997). Math1 is essential for genesis of cerebellar granule neurons. Nature 390, 169-172. doi: 10.1038/36579 
Bermingham, N. A., Hassan, B. A., Wang, V. Y., Fernandez, M., Banfi, S., Bellen, H. J., et al. (2001). Proprioceptor Pathway Development Is Dependent on MATH1. Neuron 30, 411-422.

Bloch-Gallego, E., Causeret, F., Ezan, F., Backer, S., and Hidalgo-Sánchez, M. (2005). Development of precerebellar nuclei: instructive factors and intracellular mediators in neuronal migration, survival and axon pathfinding. Brain Res. Rev. 49, 253-266. doi: 10.1016/J.BRAINRESREV.2005.01.003

Bochorishvili, G., Stornetta, R., Coates, M., and Guyenet, P. (2012). Pre-Bötzinger complex receives glutamatergic innervation from galaninergic and other retrotrapezoid nucleus neurons. J. Comp. Neurol. 520, 1047-1061. doi: 10.1002/ CNE.22769

Bourrat, F., and Sotelo, C. (1990). Migratory pathways and selective aggregation of the lateral reticular neurons in the rat embryo: a horseradish peroxidase in vitro study, with special reference to migration patterns of the precerebellar nuclei. J. Comp. Neurol. 294, 1-13. doi: 10.1002/CNE.902940102

Bouvier, J., Thoby-Brisson, M., Renier, N., Dubreuil, V., Ericson, J., Champagnat, J., et al. (2010). Hindbrain interneurons and axon guidance signaling critical for breathing. Nat. Neurosci. 13, 1066-1074. doi: 10.1038/NN.2622

Briscoe, J., Sussel, L., Serup, P., Hartigan-O’Connor, D., Jessell, T. M., Rubenstein, J. L. R., et al. (1999). Homeobox gene Nkx2.2 and specification of neuronal identity by graded Sonic hedgehog signalling. Nature 398, 622-627. doi: 10 . $1038 / 19315$

Briscoe, J., and Wilkinson, D. G. (2004). Establishing neuronal circuitry: Hox genes make the connection. Genes Dev. 18, 1643-1648. doi: 10.1101/GAD.1227004

Bruinstroop, E., Cano, G., Vanderhorst, V., Cavalcante, J., Wirth, J., Sena-Esteves, M., et al. (2012). Spinal projections of the A5, A6 (locus coeruleus), and A7 noradrenergic cell groups in rats. J. Comp. Neurol. 520, 1985-2001. doi: 10.1002/ CNE. 23024

Brunet, J., and Pattyn, A. (2002). Phox2 genes - from patterning to connectivity. Curr. Opin. Genet. Dev. 12, 435-440.

Bureau, I., Shepherd, G. M. G., and Svoboda, K. (2008). Circuit and plasticity defects in the developing somatosensory cortex of Fmr1 Knock-Out Mice. J. Neurosci. 28, 5178-5188. doi: 10.1523/JNEUROSCI.1076-08.2008

Byrum, C., and Guyenet, P. (1987). Afferent and efferent connections of the A5 noradrenergic cell group in the rat. J. Comp. Neurol. 261, 529-542. doi: 10.1002/ CNE.902610406

Cambronero, F., and Puelles, L. (2000). Rostrocaudal nuclear relationships in the avian medulla oblongata: a fate map with quail chick chimeras. J. Comp. Neurol $427,522-545$.

Cant, N., and Benson, C. (2008). Organization of the inferior colliculus of the gerbil (Meriones unguiculatus): projections from the cochlear nucleus. Neuroscience 154, 206-217. doi: 10.1016/J.NEUROSCIENCE.2008.02.015

Carpenter, E. M., Goddard, J. M., Chisaka, O., Manley, N. R., and Capecchi, M. R. (1993). Loss of Hox-A1 (Hox-1.6) function results in the reorganization of the murine hindbrain. Development 118, 1063-1075. doi: 10.1242/DEV.118.4.1063

Carr, C., and Soares, D. (2002). Evolutionary convergence and shared computational principles in the auditory system. Brain Behav. Evol. 59, 294-311. doi: 10.1159/000063565

Causeret, F., Danne, F., Ezan, F., Sotelo, C., and Bloch-Gallego, E. (2002). Slit antagonizes netrin-1 attractive effects during the migration of inferior olivary neurons. Dev. Biol. 246, 429-440. doi: 10.1006/DBIO.2002.0681

Chabrat, A., Brisson, G., Doucet-Beaupré, H., Salesse, C., Schaan Profes, M., Dovonou, A., et al. (2017). Transcriptional repression of Plxncl by Lmxla and Lmxlb directs topographic dopaminergic circuit formation. Nat. Commun. $8: 933$.

Chagnaud, B. P., Engelmann, J., Fritzsch, B., Glover, J. C., and Straka, H. (2017). Sensing external and self-motion with hair cells, a comparison of the lateral line and vestibular systems from a developmental and evolutionary perspective. Brain Behav. Evol. 90, 98-116. doi: 10.1159/000456646

Chambers, D., Wilson, L. J., Alfonsi, F., Hunter, E., Saxena, U., Blanc, E., et al. (2009). Rhombomere-specific analysis reveals the repertoire of genetic cues expressed across the developing hindbrain. Neural Dev. 4:6. doi: 10.1186/17498104-4-6

Champagnat, J., Morin-Surun, M.-P., Bouvier, J., Thoby-Brisson, M., and Fortin, G. (2011). Prenatal development of central rhythm generation. Respir. Physiol. Neurobiol. 178, 146-155. doi: 10.1016/J.RESP.2011.04.013

Champagnat, J., Morin-Surun, M. P., Fortin, G., and Thoby-Brisson, M. (2009). Developmental basis of the rostro-caudal organization of the brainstem respiratory rhythm generator. Philos. Trans. R. Soc. Lond. B Biol. Sci. 364, 2469-2476. doi: 10.1098/RSTB.2009.0090

Chatterjee, M., Li, K., Chen, L., Maisano, X., Guo, Q., Gan, L., et al. (2012). Gbx2 regulates thalamocortical axon guidance by modifying the LIM and Robo codes. Development 139, 4633-4643. doi: 10.1242/DEV.086991

Chédotal, A., and Richards, L. J. (2010). Wiring the brain: the biology of neuronal guidance. Cold Spring Harb. Perspect. Biol. 2:a001917. doi: 10.1101/ CSHPERSPECT.A001917

Chen, Y., Takano-Maruyama, M., Fritzsch, B., and Gaufo, G. O. (2012). Hoxb1 controls anteroposterior identity of vestibular projection neurons. PLoS One 7:e34762. doi: 10.1371/journal.pone.0034762

Cheng, H. J., Nakamoto, M., Bergemann, A. D., and Flanagan, J. G. (1995). Complementary gradients in expression and binding of ELF-1 and Mek4 in development of the topographic retinotectal projection map. Cell 82, 371-381.

Cheng, L., Arata, A., Mizuguchi, R., Qian, Y., Karunaratne, A., Gray, P. A., et al. (2004). Tlx3 and Tlx 1 are post-mitotic selector genes determining glutamatergic over GABAergic cell fates. Nat. Neurosci. 7, 510-517. doi: 10.1038/nn1221

Cheng, L., Samad, O. A., Xu, Y., Mizuguchi, R., Luo, P., Shirasawa, S., et al. (2005). Lbx1 and Tlx3 are opposing switches in determining GABAergic versus glutamatergic transmitter phenotypes. Nat. Neurosci. 8, 1510-1515. doi: 10. 1038/nn1569

Chizhikov, V. V., Iskusnykh, I. Y., Fattakhov, N., and Fritzsch, B. (2021). Lmxla and Lmx1b are redundantly required for the development of multiple components of the mammalian auditory system. Neuroscience 452, 247-264. doi: 10.1016/J. NEUROSCIENCE.2020.11.013

Chou, S., and Tole, S. (2019). Lhx2, an evolutionarily conserved, multifunctional regulator of forebrain development. Brain Res. 1705, 1-14. doi: 10.1016/J. BRAINRES.2018.02.046

Cicirata, F., Zappalà, A., Serapide, M., Parenti, R., Pantò, M., and Paz, C. (2005). Different pontine projections to the two sides of the cerebellum. Brain Res. Brain Res. Rev. 49, 280-294. doi: 10.1016/J.BRAINRESREV.2005.02.002

Clark, F. M., and Proudfit, H. K. (1993). The projections of noradrenergic neurons in the A5 catecholamine cell group to the spinal cord in the rat: anatomical evidence that A5 neurons modulate nociception. Brain Res. 616, 200-210. doi: 10.1016/0006-8993(93)90210-E

Colman, D. R., and Filbin, M. T. (1999). Cell Adhesion Molecules and Axonal Outgrowth. Available online at: https://www.ncbi.nlm.nih.gov/books/ NBK28015/ (accessed August 4, 2021).

Comer, J. D., Alvarez, S., Butler, S. J., and Kaltschmidt, J. A. (2019). Commissural axon guidance in the developing spinal cord: from Cajal to the present day. Neural Dev. 14:9.

Corson, S. L., Kim, M., Mistretta, C. M., and Bradley, R. (2013). Gustatory solitary tract development: a role for neuropilins. Neuroscience 252, 35-44. doi: 10.1016/ J.NEUROSCIENCE.2013.07.068

Cramer, K. S., Bermingham-McDonogh, O., Krull, C. E., and Rubel, E. W. (2004). EphA4 signaling promotes axon segregation in the developing auditory system. Dev. Biol. 269, 26-35. doi: 10.1016/J.YDBIO.2004.01.002

Cramer, K. S., Cerretti, D. P., and Siddiqui, S. A. (2006). EphB2 regulates axonal growth at the midline in the developing auditory brainstem. Dev. Biol. 295, 76-89. doi: 10.1016/J.YDBIO.2006.03.010

Cramer, K. S., Fraser, S. E., and Rubel, E. W. (2000). Embryonic origins of auditory brain-stem nuclei in the chick Hindbrain. Dev. Biol. 224, 138-151. doi: 10.1006/ dbio.2000.9779

Cramer, K. S., and Gabriele, M. L. (2014). Axon guidance in the auditory system: multiple functions of Eph receptors. Neuroscience 277, 152-162. doi: 10.1016/J. NEUROSCIENCE.2014.06.068

Cunningham, E. T., and Sawchenko, P. E. (2000). Dorsal medullary pathways subserving oromotor reflexes in the rat: implications for the central neural control of swallowing. J. Comp. Neurol. 417, 448-466.

Darnell, J. C., Jensen, K. B., Jin, P., Brown, V., Warren, S. T., and Darnell, R. B. (2001). Fragile X mental retardation protein targets G Quartet mRNAs important for neuronal function. Cell 107, 489-499.

Dauger, S., Pattyn, A., Lofaso, F., Gaultier, C., Goridis, C., Gallego, J., et al. (2003). Phox $2 \mathrm{~b}$ controls the development of peripheral chemoreceptors and afferent visceral pathways. Development 130, 6635-6642. doi: 10.1242/DEV.00866

D’Autréaux, F., Coppola, E., Hirsch, M.-R., Birchmeier, C., and Brunet, J.-F. (2011). Homeoprotein Phox 2 b commands a somatic-to-visceral switch in 
cranial sensory pathways. Proc. Natl. Acad. Sci. U.S.A. 108, 20018-20023. doi: 10.1073/PNAS.1110416108

Davis, J., and Broadie, K. (2017). Multifarious functions of the Fragile X Mental retardation protein. Trends Genet. 33, 703-714. doi: 10.1016/J.TIG.2017.07.008

de Diego, I., Kyriakopoulou, K., Karagogeos, D., and Wassef, M. (2002). Multiple influences on the migration of precerebellar neurons in the caudal medulla. Development 129, 297-306.

de Sousa Buck, H., Caous, C., and Lindsey, C. (2001). Projections of the paratrigeminal nucleus to the ambiguus, rostroventrolateral and lateral reticular nuclei, and the solitary tract. Auton. Neurosci. 87, 187-200. doi: 10.1016/S15660702(00)00259-9

del Toro, E. D., Borday, V., Davenne, M., Neun, R., Rijli, F. M., and Champagnat, J. (2001). Generation of a novel functional neuronal circuit in hoxal mutant mice. J. Neurosci. 21, 5637-5642. doi: 10.1523/JNEUROSCI.21-15-05637. 2001

Di Bonito, M., and Studer, M. (2017). Cellular and molecular underpinnings of neuronal assembly in the central auditory system during mouse development. Front. Neural Circuits 11:18. doi: 10.3389/FNCIR.2017.00018

Di Bonito, M., Boulland, J.-L., Krezel, W., Setti, E., Studer, M., and Glover, J. C. (2015). Loss of projections, functional compensation, and residual deficits in the mammalian vestibulospinal system of Hoxb1-Deficient mice. eNeuro 2:ENEURO.0096-15.2015. doi: 10.1523/ENEURO.0096-15.2015

Di Bonito, M., Narita, Y., Avallone, B., Sequino, L., Mancuso, M., Andolfi, G., et al. (2013). Assembly of the auditory circuitry by a Hox genetic network in the mouse brainstem. PLoS Genet. 9:e1003249. doi: 10.1371/JOURNAL.PGEN. 1003249

Di Bonito, M., Studer, M., and Puelles, L. (2017). Nuclear derivatives and axonal projections originating from rhombomere 4 in the mouse hindbrain. Brain Struct. Funct. 222, 3509-3542. doi: 10.1007/s00429-017-1416-0

Díaz, C., Glover, J. C., Puelles, L., and Bjaalie, J. G. (2003). The relationship between hodological and cytoarchitectonic organization in the vestibular complex of the 11-day chicken embryo. J. Comp. Neurol. 457, 87-105. doi: 10.1002/CNE.10528

Díaz, C., and Puelles, L. (2019). Segmental analysis of the vestibular nerve and the efferents of the vestibular complex. Anat. Rec. 302, 472-484. doi: 10.1002/AR. 23828

Díaz, C., Puelles, L., Marín, F., and Glover, J. C. (1998). The relationship between rhombomeres and vestibular neuron populations as assessed in quail-chicken chimeras. Dev. Biol. 202, 14-28. doi: 10.1006/dbio.1998.8986

Dickson, B., and Gilestro, G. (2006). Regulation of commissural axon pathfinding by slit and its Robo receptors. Annu. Rev. Cell Dev. Biol. 22, 651-675. doi: 10.1146/ANNUREV.CELLBIO.21.090704.151234

DiPietrantonio, H. J., and Dymecki, S. M. (2009). Zic1 levels regulate mossy fiber neuron position and axon laterality choice in the ventral brain stem. Neuroscience 162, 560-573. doi: 10.1016/j.neuroscience.2009.02.082

Dominici, C., Moreno-Bravo, J. A., Puiggros, S. R., Rappeneau, Q., Rama, N., Vieugue, P., et al. (2017). Floor-plate-derived netrin-1 is dispensable for commissural axon guidance. Nature 545, 350-354. doi: 10.1038/ NATURE22331

Donovan, L. J., Spencer, W. C., Kitt, M. M., Eastman, B. A., Lobur, K. J., Jiao, K., et al. (2019). Lmxlb is required at multiple stages to build expansive serotonergic axon architectures. eLife 8:e48788. doi: 10.7554/ELIFE.4 8788

Dubreuil, V., Ramanantsoa, N., Trochet, D., Vaubourg, V., Amiel, J., Gallego, J., et al. (2008). A human mutation in Phox $2 \mathrm{~b}$ causes lack of CO2 chemosensitivity, fatal central apnea, and specific loss of parafacial neurons. Proc. Natl. Acad. Sci. U.S.A. 105, 1067-1072. doi: 10.1073/pnas.0709115105

Dubreuil, V., Thoby-Brisson, M., Rallu, M., Persson, K., Pattyn, A., Birchmeier, C., et al. (2009). Defective respiratory rhythmogenesis and loss of central chemosensitivity in Phox $2 \mathrm{~b}$ mutants targeting retrotrapezoid nucleus neurons. J. Neurosci. 29, 14836-14846. doi: 10.1523/JNEUROSCI.2623-09.2009

Elliott, K. L., Pavlínková, G., Chizhikov, V. V., Yamoah, E. N., and Fritzsch, B. (2021). Development in the mammalian auditory system depends on transcription factors. Int. J. Mol. Sci. 22:4189. doi: 10.3390/IJMS2208 4189

Escalante, A., Murillo, B., Morenilla-Palao, C., Klar, A., and Herrera, E. (2013). Zic2-dependent axon midline avoidance controls the formation of major ipsilateral tracts in the CNS. Neuron 80, 1392-1406. doi: 10.1016/J.NEURON. 2013.10.007
Ezure, K., Tanaka, I., and Saito, Y. (2003). Brainstem and spinal projections of augmenting expiratory neurons in the rat. Neurosci. Res. 45, 41-51. doi: 10. 1016/S0168-0102(02)00197-9

Farago, A. F., Awatramani, R. B., and Dymecki, S. M. (2006). Assembly of the brainstem cochlear nuclear complex is revealed by intersectional and subtractive genetic fate maps. Neuron 50, 205-218. doi: 10.1016/j.neuron.2006. 03.014

Fedtsova, N., and Turner, E. (1995). Brn-3.0 expression identifies early post-mitotic CNS neurons and sensory neural precursors. Mech. Dev. 53, 291-304.

Feldman, J., and Kam, K. (2015). Facing the challenge of mammalian neural microcircuits: taking a few breaths may help. J. Physiol. 593, 3-23. doi: 10.1113/ JPHYSIOL.2014.277632

Frank, D., and Sela-Donenfeld, D. (2018). Hindbrain induction and patterning during early vertebrate development. Cell. Mol. Life Sci. 76, 941-960. doi: 10. 1007/S00018-018-2974-X

Friocourt, F., Kozulin, P., Belle, M., Suárez, R., Di-Poï, N., Richards, L., et al. (2019). Shared and differential features of Robo3 expression pattern in amniotes. J. Comp. Neurol. 527, 2009-2029. doi: 10.1002/CNE.24648

Fritschy, J., and Grzanna, R. (1990). Demonstration of two separate descending noradrenergic pathways to the rat spinal cord: evidence for an intragriseal trajectory of locus coeruleus axons in the superficial layers of the dorsal horn. J. Comp. Neurol. 291, 553-582. doi: 10.1002/CNE.902910406

Fu, C., Shi, L., Wei, Z., Yu, H., Hao, Y., Tian, Y., et al. (2019). Activation of Phox2bexpressing neurons in the nucleus tractus solitarii drives breathing in mice. J. Neurosci. 39, 2837-2846. doi: 10.1523/JNEUROSCI.2048-18.2018

Fu, Y., Tvrdik, P., Makki, N., Machold, R., Paxinos, G., and Watson, C. (2013). The interfascicular trigeminal nucleus: a precerebellar nucleus in the mouse defined by retrograde neuronal tracing and genetic fate mapping. J. Comp. Neurol. 521, 697-708. doi: 10.1002/CNE.23200

Fujiyama, T., Yamada, M., Terao, M., Terashima, T., Hioki, H., Inoue, Y. U., et al. (2009). Inhibitory and excitatory subtypes of cochlear nucleus neurons are defined by distinct bHLH transcription factors. Ptfla and Atoh1. Development 136, 2049-2058. doi: 10.1242/dev.033480

García-Frigola, C., Carreres, M., Vegar, C., Mason, C., and Herrera, E. (2008). Zic2 promotes axonal divergence at the optic chiasm midline by EphB1-dependent and -independent mechanisms. Development 135, 1833-1841. doi: 10.1242/ DEV.020693

Gasparini, S., Howland, J. M., Thatcher, A. J., and Geerling, J. C. (2020). Central afferents to the nucleus of the solitary tract in rats and mice. J. Comp. Neurol. 528, 2708-2728. doi: 10.1002/cne.24927

Gasparini, S., Resch, J. M., Narayan, S. V., Peltekian, L., Iverson, G. N., Karthik, S., et al. (2018). Aldosterone-sensitive HSD2 neurons in mice. Brain Struct. Funct. 224, 387-417. doi: 10.1007/S00429-018-1778-Y

Gaufo, G. O., Flodby, P., and Capecchi, M. R. (2000). Hoxb1 controls effectors of sonic hedgehog and Mash1 signaling pathways. Development 127, 5343-5354. doi: 10.1242/DEV.127.24.5343

Gavalas, A., Davenne, M., Lumsden, A., Chambon, P., and Rijli, F. M. (1997). Role of Hoxa-2 in axon pathfinding and rostral hindbrain patterning. Development 124, 3693-3702. doi: 10.1242/DEV.124.19.3693

Geisen, M. J., di Meglio, T., Pasqualetti, M., Ducret, S., Brunet, J.-F., Chedotal, A., et al. (2008). Hox Paralog Group 2 genes control the migration of mouse pontine neurons through Slit-Robo signaling. PLoS Biol. 6:e142. doi: 10.1371/ JOURNAL.PBIO.0060142

Gerrits, N., Epema, A., and Voogd, J. (1984). The mossy fiber projection of the nucleus reticularis tegmenti pontis to the flocculus and adjacent ventral paraflocculus in the cat. Neuroscience 11, 627-644.

Glasgow, S. M., Henke, R. M., MacDonald, R. J., Wright, C. V. E., and Johnson, J. E. (2005). Ptfla determines GABAergic over glutamatergic neuronal cell fate in the spinal cord dorsal horn. Development 132, 5461-5469. doi: 10.1242/DEV.02167

Glover, J. C. (2000). Neuroepithelial 'compartments' and the specification of vestibular projections. Prog. Brain Res. 124, 3-21.

Glover, J. C. (2020). "Development and segmental organization of first order information processing centers in the hindbrain," in The Senses: A Comprehensive Reference, eds B. Fritzsch and H. Straka (Amsterdam: Elsevier), 256-272.

Glover, J. C., Elliott, K. L., Erives, A., Chizhikov, V. V., and Fritzsch, B. (2018). Wilhelm His' lasting insights into hindbrain and cranial ganglia development and evolution. Dev. Biol. 444, S14-S24. doi: 10.1016/J.YDBIO.2018.02.001 
Gottesman-Davis, A., and Peusner, K. (2010). Identification of vestibuloocular projection neurons in the developing chicken medial vestibular nucleus. J. Neurosci. Res. 88, 290-303. doi: 10.1002/JNR.22206

Gray, P. A. (2008). Transcription factors and the genetic organization of brain stem respiratory neurons. 104, 1513-1521. doi: 10.1152/JAPPLPHYSIOL.01383.2007

Gray, P. A. (2013). Transcription factors define the neuroanatomical organization of the medullary reticular formation. Front. Neuroanat. 7:7. doi: 10.3389/ FNANA.2013.00007

Gross, M. K., Dottori, M., and Goulding, M. (2002). Lbx1 specifies somatosensory association interneurons in the dorsal spinal cord. Neuron 34, 535-549.

Guthrie, S. (1997). Axon guidance: netrin receptors are revealed. Curr. Biol. 7, R6-R9. doi: 10.1016/S0960-9822(06)00007-8

Guthrie, S. (2007). Patterning and axon guidance of cranial motor neurons. Nat. Rev. Neurosci. 8, 859-871. doi: 10.1038/nrn2254

Guyenet, P. G., Bayliss, D. A., Stornetta, R. L., Fortuna, M. G., Abbott, S. B., and Depuy, S. D. (2009). Retrotrapezoid nucleus, respiratory chemosensitivity and breathing automaticity. Respir. Physiol. Neurobiol. 168, 59-68. doi: 10.1016/J. RESP.2009.02.001

Hadas, Y., Etlin, A., Falk, H., Avraham, O., Kobiler, O., Panet, A., et al. (2014). A 'tool box' for deciphering neuronal circuits in the developing chick spinal cord. Nucleic Acids Res. 42, e148. doi: 10.1093/NAR/GKU750

Hagerman, R., Berry-Kravis, E., Hazlett, H., Bailey, D., Moine, H., Kooy, R., et al. (2017). Fragile X syndrome. Nat. Rev. Dis. Primers 3:17065. doi: 10.1038/NRDP. 2017.65

Haque, F., Rancic, V., Zhang, W., Clugston, R., Ballanyi, K., and Gosgnach, S. (2018). WT1-expressing interneurons regulate left-right alternation during mammalian locomotor activity. J. Neurosci. 38, 5666-5676. doi: 10.1523/ JNEUROSCI.0328-18.2018

Hashimoto, M., and Hibi, M. (2012). Development and evolution of cerebellar neural circuits. Dev. Growth Differ. 54, 373-389. doi: 10.1111/J.1440-169X.2012. 01348.X

Heeringa, A., Wu, C., and Shore, S. (2018). Multisensory integration enhances temporal coding in ventral cochlear nucleus bushy cells. J. Neurosci. 38, 28322843. doi: 10.1523/JNEUROSCI.2244-17.2018

Helms, A. W., and Johnson, J. E. (1998). Progenitors of dorsal commissural interneurons are defined by MATH1 expression. Development 125, 919-928. doi: 10.1242/DEV.125.5.919

Helms, A. W., and Johnson, J. E. (2003). Specification of dorsal spinal cord interneurons. Curr. Opin. Neurobiol. 13, 42-49.

Herbert, H., Moga, M., and Saper, C. (1990). Connections of the parabrachial nucleus with the nucleus of the solitary tract and the medullary reticular formation in the rat. J. Comp. Neurol. 293, 540-580. doi: 10.1002/CNE. 902930404

Hernandez-Miranda, L. R., Ruffault, P.-L., Bouvier, J. C., Murray, A. J., MorinSurun, M.-P., Zampieri, N., et al. (2017). Genetic identification of a hindbrain nucleus essential for innate vocalization. Proc. Natl. Acad. Sci. U.S.A. 114, 8095-8100. doi: 10.1073/PNAS.1702893114

Hidalgo-Sánchez, M., Backer, S., Puelles, L., and Bloch-Gallego, E. (2012). Origin and plasticity of the subdivisions of the inferior olivary complex. Dev. Biol. 371, 215-226. doi: 10.1016/J.YDBIO.2012.08.019

Hirano, S., Suzuki, S., and Redies, C. (2003). The cadherin superfamily in neural development: diversity, function and interaction with other molecules. Front. Biosci. 8, d306-d355. doi: 10.2741/972

Hobert, O., and Westphal, H. (2000). Functions of LIM-homeobox genes. Trends Genet. 16, 75-83.

Hori, K., and Hoshino, M. (2012). GABAergic neuron specification in the spinal cord, the cerebellum, and the cochlear nucleus. Neural Plast. 2012:921732. doi: $10.1155 / 2012 / 921732$

Hoshino, M. (2012). Neuronal subtype specification in the cerebellum and dorsal hindbrain. Dev. Growth Differ. 54, 317-326. doi: 10.1111/j.1440-169X.2012. 01330.x

Hoshino, M., Nakamura, S., Mori, K., Kawauchi, T., Terao, M., Nishimura, Y. V., et al. (2005). Ptfla, a bHLH transcriptional gene, defines GABAergic neuronal fates in cerebellum. Neuron 47, 201-213. doi: 10.1016/j.neuron.2005. 06.007

Hoshino, M., Seto, Y., and Yamada, M. (2013). "Specification of cerebellar and precerebellar neurons," in Handbook of the Cerebellum and Cerebellar Disorders, eds M. Manto, D. Gruol, J. Schmahmann, N. Koibuchi and R. Sillitoe (Cham: Springer), 75-87. doi: 10.1007/978-94-007-1333-8_5
Howell, D. M., Morgan, W. J., Jarjour, A. A., Spirou, G. A., Berrebi, A. S., Kennedy, T. E., et al. (2007). Molecular guidance cues necessary for axon pathfinding from the ventral cochlear nucleus. J. Comp. Neurol. 504, 533-549. doi: 10.1002/CNE. 21443

Hsieh, C. Y., Nakamura, P. A., Luk, S. O., Miko, I. J., Henkemeyer, M., and Cramer, K. S. (2010). Ephrin-B reverse signaling is required for formation of strictly contralateral auditory brainstem pathways. J. Neurosci. 30, 9840-9849. doi: 10.1523/JNEUROSCI.0386-10.2010

Huang, W., Tupal, S., Huang, T., Ward, C., Neul, J., Klisch, T., et al. (2012). Atohl governs the migration of postmitotic neurons that shape respiratory effectiveness at birth and chemoresponsiveness in adulthood. Neuron 75, 799809. doi: 10.1016/J.NEURON.2012.06.027

Huffman, K., and Cramer, K. (2007). EphA4 misexpression alters tonotopic projections in the auditory brainstem. Dev. Neurobiol. 67, 1655-1668. doi: 10.1002/DNEU.20535

Ikeda, K., Kawakami, K., Onimaru, H., Okada, Y., Yokota, S., Koshiya, N., et al. (2017). The respiratory control mechanisms in the brainstem and spinal cord: integrative views of the neuroanatomy and neurophysiology. J. Physiol. Sci. 67, 45-62. doi: 10.1007/S12576-016-0475-Y

Ikeda, K., Takahashi, M., Sato, S., Igarashi, H., Ishizuka, T., Yawo, H., et al. (2015). A Phox2b BAC transgenic rat line useful for understanding respiratory rhythm generator neural circuitry. PLoS One 10:e0132475. doi: 10.1371/JOURNAL. PONE.0132475

Iskusnykh, I., Steshina, E., and Chizhikov, V. (2016). Loss of Ptfla Leads to a Widespread Cell-Fate misspecification in the brainstem, affecting the development of somatosensory and viscerosensory nuclei. J. Neurosci. 36, 2691-2710. doi: 10.1523/JNEUROSCI.2526-15.2016

Itoh, T., Takeuchi, M., Sakagami, M., Asakawa, K., Sumiyama, K., Kawakami, K., et al. (2020). Gsx2 is required for specification of neurons in the inferior olivary nuclei from Ptfla-expressing neural progenitors in zebrafish. Development 147:dev190603. doi: 10.1242/DEV.190603

Jacquin, T. D., Borday, V., Schneider-Maunoury, S., Topilko, P., Ghilini, G., Kato, F., et al. (1996). Reorganization of pontine rhythmogenic neuronal networks in Krox-20 Knockout Mice. Neuron 17, 747-758.

Jain, S., and Welshhans, K. (2016). Netrin-1 induces local translation of down syndrome cell adhesion molecule in axonal growth cones. Dev. Neurobiol. 76, 799-816. doi: 10.1002/DNEU.22360

Jen, J., Chan, W., Bosley, T., Wan, J., Carr, J., Rüb, U., et al. (2004). Mutations in a human ROBO gene disrupt hindbrain axon pathway crossing and morphogenesis. Science 304, 1509-1513. doi: 10.1126/SCIENCE.1096437

Joyner, A. L., and Zervas, M. (2006). Genetic inducible fate mapping in mouse: establishing genetic lineages and defining genetic neuroanatomy in the nervous system. Dev. Dyn. 235, 2376-2385. doi: 10.1002/DVDY.20884

Kalinovsky, A., Boukhtouche, F., Blazeski, R., Bornmann, C., Suzuki, N., Mason, C. A., et al. (2011). Development of axon-target specificity of pontocerebellar afferents. PLoS Biol. 9:e1001013. doi: 10.1371/JOURNAL.PBIO.100 1013

Kang, B., Chang, D., Mackay, D., West, G., Moreira, T., Takakura, A., et al. (2007). Central nervous system distribution of the transcription factor Phox $2 b$ in the adult rat. J. Comp. Neurol. 503, 627-641. doi: 10.1002/CNE.21409

Kang, H., Zhao, J., Jiang, X., Li, G., Huang, W., Cheng, H., et al. (2019). Drosophila Netrin-B controls mushroom body axon extension and regulates courtshipassociated learning and memory of a Drosophila fragile X syndrome model. Mol. Brain 12:52.

Kania, A., Johnson, R. L., and Jessell, T. M. (2000). Coordinate Roles for LIM homeobox genes in directing the dorsoventral trajectory of motor axons in the vertebrate limb. Cell 102, 161-173.

Karimnamazi, H., Travers, S., and Travers, J. (2002). Oral and gastric input to the parabrachial nucleus of the rat. Brain Res. 957, 193-206.

Kawauchi, D., Taniguchi, H., Watanabe, H., Saito, T., and Murakami, F. (2006). Direct visualization of nucleogenesis by precerebellar neurons: involvement of ventricle-directed, radial fibre-associated migration. Development 133, 11131123. doi: 10.1242/DEV.02283

Kayam, G., Kohl, A., Magen, Z., Peretz, Y., Weisinger, K., Bar, A., et al. (2013). A novel role for Pax6 in the segmental organization of the hindbrain. Development 140, 2190-2202. doi: 10.1242/dev.089136

Keino-Masu, K., Masu, M., Hinck, L., Leonardo, E. D., Chan, S. S. Y., Culotti, J. G., et al. (1996). Deleted in Colorectal Cancer (DCC) encodes a netrin receptor. Cell 87, 175-185. 
Kiecker, C., and Lumsden, A. (2005). Compartments and their boundaries in vertebrate brain development. Nat. Rev. Neurosci. 6, 553-564. doi: 10.1038/ nrn 1702

Kil, J., Kageyama, G., Semple, M., and Kitzes, L. (1995). Development of ventral cochlear nucleus projections to the superior olivary complex in gerbil. J. Comp. Neurol. 353, 317-340. doi: 10.1002/CNE.903530302

Kim, E. J., Battiste, J., Nakagawa, Y., and Johnson, J. E. (2008). Ascl1 (Mash1) lineage cells contribute to discrete cell populations in CNS architecture. Mol. Cell. Neurosci. 38, 595-606. doi: 10.1016/J.MCN.2008.05.008

Klein, R., and Kania, A. (2014). Ephrin signalling in the developing nervous system. Curr. Opin. Neurobiol. 27, 16-24. doi: 10.1016/J.CONB.2014.02.006

Kohl, A., Hadas, Y., Klar, A., and Sela-Donenfeld, D. (2012). Axonal patterns and targets of dA1 interneurons in the chick hindbrain. J. Neurosci. 32, 5757-5771. doi: 10.1523/JNEUROSCI.4231-11.2012

Kohl, A., Hadas, Y., Klar, A., and Sela-Donenfeld, D. (2013). Electroporation of the hindbrain to trace axonal trajectories and synaptic targets in the chick embryo. J. Vis. Exp. 75:50136. doi: 10.3791/50136

Kohl, A., Marquardt, T., Klar, A., and Sela-Donenfeld, D. (2015). Control of axon guidance and neurotransmitter phenotype of $\mathrm{dB} 1$ hindbrain interneurons by Lim-HD code. J. Neurosci. 35, 2596-2611. doi: 10.1523/JNEUROSCI.2699-14. 2015

Korematsu, K., and Redies, C. (1997). Expression of Cadherin-8 mRNA in the developing mouse central nervous system. J. Comp. Neurol. 387, 291-306.

Köster, R. W., and Fraser, S. E. (2001). Direct imaging of in vivo neuronal migration in the developing cerebellum. Curr. Biol. 11, 1858-1863.

Kratochwil, C. F., Maheshwari, U., and Rijli, F. M. (2017). The long journey of pontine nuclei neurons: from rhombic lip to cortico-ponto-cerebellar circuitry. Front. Neural Circuits 11:33. doi: 10.3389/FNCIR.2017.00033

Krützfeldt, N., Logerot, P., Kubke, M., and Wild, J. (2010b). Connections of the auditory brainstem in a songbird, Taeniopygia guttata. II. Projections of nucleus angularis and nucleus laminaris to the superior olive and lateral lemniscal nuclei. J. Comp. Neurol. 518, 2135-2148. doi: 10.1002/CNE.22324

Krützfeldt, N., Logerot, P., Kubke, M., and Wild, J. (2010a). Connections of the auditory brainstem in a songbird, Taeniopygia guttata. I. Projections of nucleus angularis and nucleus laminaris to the auditory torus. J. Comp. Neurol. 518, 2109-2134. doi: 10.1002/CNE.22334

Kuwako, K., Nishimoto, Y., Kawase, S., Okano, H., and Okano, H. (2014). Cadherin-7 regulates mossy fiber connectivity in the cerebellum. Cell Rep. 9, 311-323. doi: 10.1016/J.CELREP.2014.08.063

Landsberg, R. L., Awatramani, R. B., Hunter, N. L., Farago, A. F., DiPietrantonio, H. J., Rodriguez, C. I., et al. (2005). Hindbrain rhombic lip is comprised of discrete progenitor cell populations allocated by Pax6. Neuron 48, 933-947. doi: 10.1016/J.NEURON.2005.11.031

Lee, J., Ray, R., and Chien, C. (2001). Cloning and expression of three zebrafish roundabout homologs suggest roles in axon guidance and cell migration. Dev. Dyn. 221, 216-230. doi: 10.1002/DVDY.1136

Lee, K. J., Dietrich, P., and Jessell, T. M. (2000). Genetic Ablation Reveals that the Roof Plate is Essential for Dorsal Interneuron Specification. Available online at: www.nature.com (accessed August 1, 2021).

Lee, K. J., Mendelsohn, M., and Jessell, T. M. (1998). Neuronal patterning by BMPs: a requirement for GDF7 in the generation of a discrete class of commissural interneurons in the mouse spinal cord. Genes Dev. 12, 3394-3407.

Lee, R., Petros, T. J., and Mason, C. A. (2008). Zic2 regulates retinal ganglion cell axon avoidance of ephrinB2 through inducing expression of the guidance receptor EphB1. J. Neurosci. 28, 5910-5919. doi: 10.1523/JNEUROSCI.0632-08. 2008

Liang, H., Bácskai, T., Watson, C., and Paxinos, G. (2014). Projections from the lateral vestibular nucleus to the spinal cord in the mouse. Brain Struct. Funct. 219, 805-815.

Liem, K. F., Tremml, G., and Jessell, T. M. (1997). A role for the roof plate and its resident TGFbeta-related proteins in neuronal patterning in the dorsal spinal cord. Cell 91, 127-138.

Lihua, Q., Ahn, K. J., Lee, L. W., de Charleroy, C. J., and Crenshaw, E. B. I. (2019). Analyses with double knockouts of the Bmprla and Bmprlb genes demonstrate that BMP signaling is involved in the formation of precerebellar mossy fiber nuclei derived from the rhombic lip. PLoS One 14:e226602. doi: 10.1371/JOURNAL.PONE.0226602
Lipovsek, M., and Wingate, R. J. T. (2018). Conserved and divergent development of brainstem vestibular and auditory nuclei. eLife 7:e40232. doi: 10.7554/ELIFE. 40232

Logan, C., Wingate, R. J. T., McKay, I. J., and Lumsden, A. (1998). Tlx-1 and Tlx3 Homeobox gene expression in cranial sensory ganglia and hindbrain of the chick embryo: markers of patterned connectivity. J. Neurosci. 18, 5389-5402. doi: 10.1523/JNEUROSCI.18-14-05389.1998

Lumsden, A., and Keynes, R. (1989). Segmental patterns of neuronal development in the chick hindbrain. Nature 337, 424-428. doi: 10.1038/337424a0

Lumsden, A., and Krumlauf, R. (1996). Patterning the vertebrate neuraxis. Science 274, 1109-1115. doi: 10.1126/SCIENCE.274.5290.1109

Lunde, A., Okaty, B. W., Dymecki, S. M., and Glover, J. C. (2019). Molecular profiling defines evolutionarily conserved transcription factor signatures of major vestibulospinal neuron groups. eNeuro 6:ENEURO.0475-18.2019. doi: 10.1523/ENEURO.0475-18.2019

Lustig, M., Erskine, L., Mason, C., Grumet, M., and Sakurai, T. (2001). Nr-CAM expression in the developing mouse nervous system: ventral midline structures, specific fiber tracts, and neuropilar regions. J. Comp. Neurol. 434, 13-28. doi: 10.1002/CNE.1161

Machold, R., and Fishell, G. (2005). Math1 is expressed in temporally discrete pools of cerebellar rhombic-lip neural progenitors. Neuron 48, 17-24. doi: 10.1016/J. NEURON.2005.08.028

Machold, R., Klein, C., and Fishell, G. (2011). Genes expressed in Atoh1 neuronal lineages arising from the r1/isthmus rhombic lip. Gene Expr. Patterns 11, 349-359. doi: 10.1016/J.GEP.2011.03.007

Maklad, A., and Fritzsch, B. (2003). Development of vestibular afferent projections into the hindbrain and their central targets. Brain Res. Bull. 60, 497-510.

Maricich, S. M., Xia, A., Mathes, E. L., Wang, V. Y., Oghalai, J. S., Fritzsch, B. F., et al. (2009). Atoh1-lineal neurons are required for hearing and for the survival of neurons in the spiral ganglion and brainstem accessory auditory nuclei. J. Neurosci. 29, 11123-11133. doi: 10.1523/JNEUROSCI.2232-09.2009

Marillat, V., Sabatier, C., Failli, V., Matsunaga, E., Sotelo, C., Tessier-Lavigne, M., et al. (2004). The slit receptor Rig-1/Robo3 controls midline crossing by hindbrain precerebellar neurons and axons. Neuron 43, 69-79. doi: 10.1016/J. NEURON.2004.06.018

Marín, F., Aroca, P., and Puelles, L. (2008). Hox gene colinear expression in the avian medulla oblongata is correlated with pseudorhombomeric domains. Dev. Biol. 323, 230-247. doi: 10.1016/j.ydbio.2008.08.017

Marín, F., and Puelles, L. (1995). Morphological fate of rhombomeres in quail/chick chimeras: a segmental analysis of hindbrain nuclei. Eur. J. Neurosci. 7, 1714-1738. doi: 10.1111/J.1460-9568.1995.TB00693.X

Marin, F., Puelles, L., Marín, F., and Puelles, L. (1995). Morphological fate of rhombomeres in quail/chick chimeras: a segmental analysis of hindbrain nuclei. Eur. J. Neurosci. 7, 1714-1738.

McClintock, J. M., Kheirbek, M. A., and Prince, V. E. (2002). Knockdown of duplicated zebrafish hoxb1 genes reveals distinct roles in hindbrain patterning and a novel mechanism of duplicate gene retention. Development 129, 23392354. doi: 10.1242/DEV.129.10.2339

Mejías-Aponte, C., Drouin, C., and Aston-Jones, G. (2009). Adrenergic and noradrenergic innervation of the midbrain ventral tegmental area and retrorubral field: prominent inputs from medullary homeostatic centers. J. Neurosci. 29, 3613-3626. doi: 10.1523/JNEUROSCI.4632-08.2009

Meléndez-Herrera, E., and Varela-Echavarría, A. (2006). Expression of secreted semaphorins and their receptors in specific neuromeres, boundaries, and neuronal groups in the developing mouse and chick brain. Brain Res. 1067, 126-137. doi: 10.1016/J.BRAINRES.2005.10.028

Meredith, D. M., Masui, T., Swift, G. H., MacDonald, R. J., and Johnson, J. E. (2009). Multiple transcriptional mechanisms control Ptfla levels during neural development including autoregulation by the PTF1-J Complex. J. Neurosci. 29, 11139-11148. doi: 10.1523/JNEUROSCI.2303-09.2009

Milinkeviciute, G., and Cramer, K. S. (2020). "Development of the ascending auditory pathway," in The Senses: A Comprehensive Reference, eds B. Fritzsch and B. Grothe (Amsterdam: Elsevier), 337-353.

Mirza, R., Kivrak, B., and Erzurumlu, R. (2013). Cooperative slit and netrin signaling in contralateralization of the mouse trigeminothalamic pathway. J. Comp. Neurol. 521, 312-325. doi: 10.1002/CNE.23188

Mishima, Y., Lindgren, A. G., Chizhikov, V. V., Johnson, R. L., and Millen, K. J. (2009). Overlapping function of Lmxla and Lmxlb in anterior hindbrain roof 
plate formation and cerebellar growth. J. Neurosci. 29, 11377-11384. doi: 10. 1523/JNEUROSCI.0969-09.2009

Mizuguchi, R., Kriks, S., Cordes, R., Gossler, A., Ma, Q., and Goulding, M. (2006). Ascl1 and Gsh1/2 control inhibitory and excitatory cell fate in spinal sensory interneurons. Nat. Neurosci. 9, 770-778. doi: 10.1038/NN1706

Moens, C. B., and Prince, V. E. (2002). Constructing the hindbrain: insights from the zebrafish. Dev. Dyn. 224, 1-17. doi: 10.1002/DVDY.10086

Moore, J. K. (2000). Organization of the human superior olivary complex - Moore - 2000 - Microscopy Research and Technique - Wiley Online Library. Microsc. Res. Tech. 51, 403-412.

Moreno-Bravo, J. A., Perez-Balaguer, A., Martinez-Lopez, J. E., Aroca, P., Puelles, L., Martinez, S., et al. (2014). Role of Shh in the development of molecularly characterized tegmental nuclei in mouse rhombomere 1. Brain Struct. Funct. 219, 777-792.

Moreno-Bravo, J. A., Roig Puiggros, S., Blockus, H., Dominici, C., Zelina, P., Mehlen, P., et al. (2018). Commissural neurons transgress the CNS/PNS boundary in absence of ventricular zone-derived netrin 1. Development 145:dev159400. doi: 10.1242/DEV.159400

Mtui, E., Anwar, M., Gomez, R., Reis, D., and Ruggiero, D. (1993). Projections from the nucleus tractus solitarii to the spinal cord. J. Comp. Neurol. 337, 231-252. doi: 10.1002/CNE.903370205

Müller, T., Anlag, K., Wildner, H., Britsch, S., Treier, M., and Birchmeier, C. (2005). The bHLH factor Olig3 coordinates the specification of dorsal neurons in the spinal cord. Genes Dev. 19, 733-743. doi: 10.1101/GAD.326105

Müller, T., Brohmann, H., Pierani, A., Heppenstall, P. A., Lewin, G. R., Jessell, T. M., et al. (2002). The Homeodomain Factor Lbxl distinguishes two major programs of neuronal differentiation in the dorsal spinal cord. Neuron 34, 551-562. doi: 10.1016/S0896-6273(02)00689-X

Narita, Y., and Rijli, F. M. (2009). Hox genes in neural patterning and circuit formation in the mouse hindbrain. Curr. Top. Dev. Biol. 88, 139-167.

Neudert, F., and Redies, C. (2008). Neural circuits revealed by axon tracing and mapping cadherin expression in the embryonic chicken cerebellum. J. Comp. Neurol. 509, 283-301. doi: 10.1002/CNE.21743

Neufeld, G., Cohen, T., Shraga, N., Lange, T., Kessler, O., and Herzog, Y. (2002). The Neuropilins: multifunctional Semaphorin and VEGF Receptors that Modulate Axon Guidance and Angiogenesis. Trends Cardiovasc. Med. 12, 13-19.

Nishida, K., Flanagan, J. G., and Nakamoto, M. (2002). Domain-specific olivocerebellar projection regulated by the EphA-ephrin-A interaction. Development 129, 5647-5658. doi: 10.1242/DEV.00162

Norgren, R. (1978). Projections from the nucleus of the solitary tract in the rat. Neuroscience 3, 207-218. doi: 10.1016/0306-4522(78)90102-1

Nothwang, H. G. (2016). Evolution of mammalian sound localization circuits: a developmental perspective. Prog. Neurobiol. 141, 1-24. doi: 10.1016/J. PNEUROBIO.2016.02.003

Nothwang, H. G., Ebbers, L., Schlüter, T., and Willaredt, M. A. (2015). The emerging framework of mammalian auditory hindbrain development. Cell Tissue Res. 361, 33-48.

Okabe, N., Shimizu, K., Ozaki-Kuroda, K., Nakanishi, H., Morimoto, K., Takeuchi, M., et al. (2004). Contacts between the commissural axons and the floor plate cells are mediated by nectins. Dev. Biol. 273, 244-256. doi: 10.1016/J.YDBIO. 2004.05.034

Okada, T., Keino-Masu, K., and Masu, M. (2007). Migration and nucleogenesis of mouse precerebellar neurons visualized by in utero electroporation of a green fluorescent protein gene. Neurosci. Res. 57, 40-49. doi: 10.1016/J.NEURES.2006. 09.010

Oury, F., Murakami, Y., Renaud, J., Pasqualetti, M., Charnay, P., Ren, S., et al. (2006). Hoxa2- and rhombomere-dependent development of the mouse facial somatosensory map. Science 313, 1408-1413. doi: 10.1126/SCIENCE. 1130042

Overholt, E., Rubel, E., and Hyson, R. (1992). A circuit for coding interaural time differences in the chick brainstem. J. Neurosci. 12, 1698-1708. doi: 10.1523/ JNEUROSCI.12-05-01698.1992

Pagliardini, S., Ren, J. R., Gray, P. A., VanDunk, C., Gross, M., Goulding, M., et al. (2008). Central Respiratory Rhythmogenesis Is Abnormal in Lbx1- Deficient Mice. J. Neurosci. 28, 11030-11041. doi: 10.1523/JNEUROSCI.1648-08.2008

Palmesino, E., Rousso, D. L., Kao, T.-J., Klar, A., Laufer, E., Uemura, O., et al. (2010). Foxp1 and Lhx1 coordinate motor neuron migration with axon trajectory choice by gating reelin signalling. PLoS Biol. 8:e1000446. doi: 10.1371/ JOURNAL.PBIO.1000446

Paradies, M., and Eisenman, L. (1993). Evidence of early topographic organization in the embryonic olivocerebellar projection: a model system for the study of pattern formation processes in the central nervous system. Dev. Dyn. 197, 125-145. doi: 10.1002/AJA.1001970206

Parker, H. J., and Krumlauf, R. (2020). A Hox gene regulatory network for hindbrain segmentation. Curr. Top. Dev. Biol. 139, 169-203. doi: 10.1016/BS. CTDB.2020.03.001

Pasqualetti, M., Diaz, C., Renaud, J.-S., Rijli, F. M., and Glover, J. C. (2007). FateMapping the mammalian hindbrain: segmental origins of vestibular projection neurons assessed using Rhombomere-Specific Hoxa2 enhancer elements in the mouse embryo. J. Neurosci. 27, 9670-9681. doi: 10.1523/JNEUROSCI.2189-07. 2007

Pasterkamp, R. J. (2012). Getting neural circuits into shape with semaphorins. Nat. Rev. Neurosci. 13, 605-618. doi: 10.1038/nrn3302

Pattyn, A., Guillemot, F., and Brunet, J. (2006). Delays in neuronal differentiation in Mash1/Ascll mutants. Dev. Biol. 295, 67-75. doi: 10.1016/J.YDBIO.2006. 03.008

Pillai, A., Mansouri, A., Behringer, R., Westphal, H., and Goulding, M. (2007). Lhx1 and Lhx 5 maintain the inhibitory-neurotransmitter status of interneurons in the dorsal spinal cord. Development 134, 357-366. doi: 10.1242/DEV.02717

Pinto, M., Olyntho-Tokunaga, H., Souccar, C., Schoorlemmer, G., and Lapa Rde, C. (2007). The interstitial system of the trigeminal spinal tract projects to the red nucleus in mice. Somatosens. Mot. Res. 24, 221-225. doi: 10.1080/ 08990220701802950

Pollerberg, G. E., Thelen, K., Theiss, M. O., and Hochlehnert, B. C. (2013). The role of cell adhesion molecules for navigating axons: density matters. Mech. Dev. 130, 359-372. doi: 10.1016/J.MOD.2012.11.002

Price, C., Hoyda, T., and Ferguson, A. (2008). The area postrema: a brain monitor and integrator of systemic autonomic state. Neuroscientist 14, 182-194. doi: $10.1177 / 1073858407311100$

Puelles, L. (2013). "Plan of the developing vertebrate nervous system: relating embryology to the adult nervous system (Prosomere Model, Overview of Brain Organization)," in Patterning and Cell Type Specification in the Developing CNS and PNS, eds J. Rubenstein and P. Rakic (Amsterdam: Academic Press), 187-209. doi: 10.1016/B978-0-12-397265-1.00118-0

Puelles, L., Harrison, M., Paxinos, G., and Watson, C. (2013). A developmental ontology for the mammalian brain based on the prosomeric model. Trends Neurosci. 36, 570-578. doi: 10.1016/j.tins.2013.06.004

Puelles, L., Robles, C., Martínez-de-la-Torre, M., and Martínez, S. (1994). New subdivision schema for the avian torus semicircularis: neurochemical maps in the chick. J. Comp. Neurol. 340, 98-125. doi: 10.1002/cne.903400108

Qian, Y., Fritzsch, B., Shirasawa, S., Chen, C.-L., Choi, Y., and Ma, Q. (2001). Formation of brainstem (nor)adrenergic centers and first-order relay visceral sensory neurons is dependent on homeodomain protein $\mathrm{Rnx} / \mathrm{Tlx}$ 3. Genes Dev. 15, 2533-2545. doi: 10.1101/GAD.921501

Qian, Y., Shirasawa, S., Chen, C.-L., Cheng, L., and Ma, Q. (2002). Proper development of relay somatic sensory neurons and D2/D4 interneurons requires homeobox genes Rnx/Tlx-3 and Tlx-1. Genes Dev. 16, 1220-1233. doi: $10.1101 /$ gad. 982802

Ray, R., and Dymecki, S. (2009). Rautenlippe Redux - toward a unified view of the precerebellar rhombic lip. Curr. Opin. Cell Biol. 21, 741-747. doi: 10.1016/J. CEB.2009.10.003

Redies, C., and Takeichi, M. (1993). Expression of N-cadherin mRNA during development of the mouse brain. Dev. Dyn. 197, 26-39. doi: 10.1002/AJA. 1001970104

Reeber, S., White, J., George-Jones, N., and Sillitoe, R. (2013). Architecture and development of olivocerebellar circuit topography. Front. Neural Circuits 6:115. doi: 10.3389/FNCIR.2012.00115

Renier, N., Schonewille, M., Giraudet, F., Badura, A., Tessier-Lavigne, M., Avan, P., et al. (2010). Genetic dissection of the function of hindbrain axonal commissures. PLoS Biol. 8:e1000325. doi: 10.1371/JOURNAL.PBIO.1000325

Rodriguez, C. I., and Dymecki, S. M. (2000). Origin of the precerebellar system. Neuron 27, 475-486.

Rose, M. F., Ahmad, K. A., Thaller, C., and Zoghbi, H. Y. (2009a). Excitatory neurons of the proprioceptive, interoceptive, and arousal hindbrain networks 
share a developmental requirement for Math1. Proc. Natl. Acad. Sci. U.S.A. 106, 22462-22467. doi: 10.1073/pnas.0911579106

Rose, M. F., Ren, J., Ahmad, K. A., Chao, H. T., Klisch, T. J., Flora, A., et al. (2009b). Math1 is essential for the development of hindbrain neurons critical for perinatal breathing. Neuron 64, 341-354. doi: 10.1016/j.neuron.2009.10. 023

Roy, A., Francius, C., Rousso, D., Seuntjens, E., Debruyn, J., Luxenhofer, G., et al. (2012). Onecut transcription factors act upstream of Isl1 to regulate spinal motoneuron diversification. Development 139, 3109-3119. doi: 10.1242/DEV. 078501

Rubel, E. W., and Parks, T. N. (1975). Organization and development of brain stem auditory nuclei of the chicken: tonotopic organization of N. magnocellularis and N. laminaris. J. Comp. Neurol. 164, 411-433. doi: 10.1002/CNE.901640403

Rubel, E. W., and Parks, T. N. (1988). "Organization and development of the avian brainstem auditory system," in Auditory Function: Neurobiological Bases of Hearing, eds G. M. Edelman, W. G. Einar, and W. M. Cowan (New York, NY: John Wiley), 3-92.

Ryugo, D. K., and Parks, T. N. (2003). Primary innervation of the avian and mammalian cochlear nucleus. Brain Res. Bull. 60, $435-456$.

Sakai, N., Insolera, R., Sillitoe, R. V., Shi, S.-H., and Kaprielian, Z. (2012). Axon Sorting within the Spinal Cord Marginal Zone via Robo-Mediated Inhibition of N-Cadherin Controls Spinocerebellar Tract Formation. J. Neurosci 32, 1537715387. doi: 10.1523/JNEUROSCI.2225-12.2012

Sawada, K., Fukui, Y., and Hawkes, R. (2008). Spatial distribution of corticotropinreleasing factor immunopositive climbing fibers in the mouse cerebellum: analysis by whole mount immunohistochemistry. Brain Res. 1222, 106-117. doi: 10.1016/J.BRAINRES.2008.05.029

Sawtell, N. B., and Bell, C. C. (2013). "Cerebellum-like structures," in Handbook of the Cerebellum and Cerebellar Disorders, eds M. Manto, J. D. Schmahmann, F. Rossi, D. L. Gruol and N. Koibuchi (Dordrecht: Springer), 1257-1277. doi: 10.1007/978-94-007-1333-8_55

Scharkowski, F., Frotscher, M., Lutz, D., Korte, M., and Michaelsen-Preusse, K. (2018). Altered connectivity and synapse maturation of the hippocampal mossy fiber pathway in a mouse model of the Fragile X Syndrome. Cereb. Cortex 28, 852-867. doi: 10.1093/CERCOR/BHW408

Schinzel, F., Seyfer, H., Ebbers, L., and Nothwang, H. (2021). The Lbx1 lineage differentially contributes to inhibitory cell types of the dorsal cochlear nucleus, a cerebellum-like structure, and the cerebellum. J. Comp. Neurol. 529, 3032-3045. doi: 10.1002/CNE.25147

Schnerwitzki, D., Hayn, C., Perner, B., and Englert, C. (2020). Wt1 positive dB4 neurons in the hindbrain are crucial for respiration. Front. Neurosci. 14:529487. doi: 10.3389/FNINS.2020.529487

Schnerwitzki, D., Perry, S., Ivanova, A., Caixeta, F., Cramer, P., Günther, S., et al. (2018). Neuron-specific inactivation of Wt1 alters locomotion in mice and changes interneuron composition in the spinal cord. Life Sci. Alliance 1:e201800106. doi: 10.26508/LSA.201800106

Schubert, F. R., Dietrich, S., Mootoosamy, R. C., Chapman, S. C., and Lumsden, A. (2001). Lbxl marks a subset of interneurons in chick hindbrain and spinal cord. Mech. Dev. 101, 181-185.

Seidl, A. H., Sanchez, J. T., Schecterson, L., Tabor, K. M., Wang, Y., Kashima, D. T., et al. (2013). Transgenic quail as a model for research in the avian nervous system: a comparative study of the auditory brainstem. J. Comp. Neurol. 521, 5-23. doi: $10.1002 / \mathrm{cne} .23187$

Shamay-Ramot, A., Khermesh, K., Porath, H. T., Barak, M., Pinto, Y., Wachtel, C., et al. (2015). Fmrp interacts with adar and regulates RNA editing, synaptic density and locomotor activity in Zebrafish. PLoS Genet. 11:e1005702. doi: 10.1371/JOURNAL.PGEN.1005702

Shapiro, R., and Miselis, R. (1985). The central neural connections of the area postrema of the rat. J. Comp. Neurol. 234, 344-364. doi: 10.1002/CNE. 902340306

Sharma, P. M., Yang, X., Bowman, M., Roberts, V., and Sukumar, S. (1992). Molecular cloning of Rat Wilms' tumor complementary DNA and a Study of Messenger RNA Expression in the Urogenital System and the Brain. Cancer Res. 52, 6407-6412.

Shimamura, K., Takahashi, T., and Takeichi, M. (1992). E-cadherin expression in a particular subset of sensory neurons. Dev. Biol. 152, 242-254. doi: 10.1016/ 0012-1606(92)90132-Z
Shirasaki, R., and Pfaff, S. L. (2003). Transcriptional codes and the control of neuronal identity. Annu. Rev. Neurosci. 25, 251-281. doi: 10.1146/ANNUREV. NEURO.25.112701.142916

Shoja-Taheri, F., DeMarco, A., and Mastick, G. S. (2015). Netrin1-DCC-mediated attraction guides post-crossing commissural axons in the hindbrain. J. Neurosci. 35, 11707-11718. doi: 10.1523/JNEUROSCI.0613-15.2015

Shoji, H., Ito, T., Wakamatsu, Y., Hayasaka, N., Ohsaki, K., Oyanagi, M., et al. (1996). Regionalized expression of the Dbx family homeobox genes in the embryonic CNS of the mouse. Mech. Dev. 56, 25-39.

Sieber, M. A., Storm, R., Martinez-de-la-Torre, M., Müller, T., Wende, H., Reuter, K., et al. (2007). Lbx1 acts as a selector gene in the fate determination of somatosensory and viscerosensory relay neurons in the hindbrain. J. Neurosci. 27, 4902-4909. doi: 10.1523/JNEUROSCI.0717-07.2007

Smith, J. C., Abdala, A. P. L., Rybak, I. A., and Paton, J. F. R. (2009). Structural and functional architecture of respiratory networks in the mammalian brainstem. Philos. Trans. R. Soc. Lond. B Biol. Sci. 364, 2577-2587. doi: 10.1098/RSTB.2009. 0081

Soares, D., Chitwood, R., Hyson, R., and Carr, C. (2002). Intrinsic neuronal properties of the chick nucleus angularis. J. Neurophysiol. 88, 152-162. doi: 10.1152/JN.2002.88.1.152

Soares, M., Nakazawa, M., Ishikawa, K., Sato, T., and Honda, K. (2013). Hearing screening for Japanese children and young adults using the automated auditory brainstem response. Auris Nasus Larynx 41, 17-21. doi: 10.1016/j.anl.2013. 08.001

Solowska, J. M., Mazurek, A., Weinberger, L., and Baird, D. H. (2002). Pontocerebellar Axon Guidance: neuropilin-1- and Semaphorin 3A-Sensitivity Gradients across Basilar Pontine Nuclei and Semaphorin 3A Variation across Cerebellum. Mol. Cell. Neurosci. 21, 266-284. doi: 10.1006/MCNE.2002.1187

Stein, M. K., and Loewy, A. D. (2010). Area Postrema Projects to FoxP2 neurons of the Pre-Locus Coeruleus and Parabrachial Nuclei: brainstem sites implicated in sodium appetite regulation. Brain Res. 1359, 116-127. doi: 10.1016/J. BRAINRES.2010.08.085

Stiles, J., and Jernigan, T. L. (2010). The basics of brain development. Neuropsychol. Rev. 20, 327-348.

Stoeckli, E. (2018). Understanding axon guidance: Are we nearly there yet? Development 145:dev151415. doi: 10.1242/DEV.151415

Stoeckli, E. T., and Landmesser, L. T. (1995). Axonin-1, Nr-CAM, and Ng-CAM play different roles in the in vivo guidance of chick commissural neurons. Neuron 14, 1165-1179.

Storm, R., Cholewa-Waclaw, J., Reuter, K., Bröhl, D., Sieber, M., Treier, M., et al. (2009). The bHLH transcription factor Olig3 marks the dorsal neuroepithelium of the hindbrain and is essential for the development of brainstem nuclei. Development 136, 295-305. doi: 10.1242/DEV.027193

Stornetta, R. L., Moreira, T. S., Takakura, A. C., Kang, B. J., Chang, D. A., West, G. H., et al. (2006). Expression of Phox $2 \mathrm{~b}$ by brainstem neurons involved in chemosensory integration in the adult rat. J. Neurosci. 26, 10305-10314. doi: 10.1523/JNEUROSCI.2917-06.2006

Straka, H., Baker, R., and Gilland, E. (2001). Rhombomeric organization of vestibular pathways in larval frogs. J. Comp. Neurol. 437, 42-55. doi: 10.1002/ CNE. 1268

Straka, H., Fritzsch, B., and Glover, J. (2014). Connecting ears to eye muscles: evolution of a "simple" reflex arc. Brain Behav. Evol. 83, 162-175. doi: 10.1159/ 000357833

Takahashi, T., and Konishi, M. (1988). Projections of nucleus angularis and nucleus laminaris to the lateral lemniscal nuclear complex of the barn owl. J. Comp. Neurol. 274, 212-238. doi: 10.1002/CNE.902740207

Tamada, A., Kumada, T., Zhu, Y., Matsumoto, T., Hatanaka, Y., Muguruma, K., et al. (2008). Crucial roles of Robo proteins in midline crossing of cerebellofugal axons and lack of their up-regulation after midline crossing. Neural Dev. 3:29. doi: 10.1186/1749-8104-3-29

Taniguchi, H., Kawauchi, D., Nishida, K., and Murakami, F. (2006). Classic cadherins regulate tangential migration of precerebellar neurons in the caudal hindbrain. Development 133, 1923-1931. doi: 10.1242/DEV.02354

Tashiro, Y., Yanagawa, Y., Obata, K., and Murakami, F. (2007). Development and migration of GABAergic neurons in the mouse myelencephalon. J. Comp. Neurol. 503, 260-269. doi: 10.1002/CNE.21380

Tessier-Lavigne, M., and Goodman, C. S. (1996). The molecular biology of axon guidance. Science 274, 1123-1133. doi: 10.1126/SCIENCE.274.5290.1123 
Thoby-Brisson, M., Karlén, M., Wu, N., Charnay, P., Champagnat, J., and Fortin, G. (2009). Genetic identification of an embryonic parafacial oscillator coupling to the preBötzinger complex. Nat. Neurosci. 12, 1028-1035. doi: 10.1038/nn. 2354

Tilleman, H., Hakim, V., Novikov, O., Liser, K., Nashelsky, L., di Salvio, M., et al. (2010). Bmp5/7 in concert with the mid-hindbrain organizer control development of noradrenergic locus coeruleus neurons. Mol. Cell. Neurosci. 45, 1-11. doi: 10.1016/J.MCN.2010.05.003

Tomás-Roca, L., Corral-San-Miguel, R., Aroca, P., Puelles, L., and Marín, F. (2016). Crypto-rhombomeres of the mouse medulla oblongata, defined by molecular and morphological features. Brain Struct. Funct. 221, 815-838. doi: 10.1007/ s00429-014-0938-y

Trainor, P. A., and Krumlauf, R. (2001). Hox genes, neural crest cells and branchial arch patterning. Curr. Opin. Cell Biol. 13, 698-705. doi: 10.1016/S0955-0674(00) 00273-8

Trussell, L. O., and Oertel, D. (2018). "Microcircuits of the dorsal cochlear nucleus," in The Mammalian Auditory Pathways. Springer Handbook of Auditory Research, Vol. 65, eds D. Oliver, N. Cant, R. Fay, and A. Popper (Cham: Springer), 73-99. doi: 10.1007/978-3-319-71798-2_4

Vallstedt, A., and Kullander, K. (2013). Dorsally derived spinal interneurons in locomotor circuits. Ann. N. Y. Acad. Sci. 1279, 32-42. doi: 10.1111/J.1749-6632. 2012.06801.X

van der Heijden, M., and Zoghbi, H. (2018). Loss of Atoh1 from neurons regulating hypoxic and hypercapnic chemoresponses causes neonatal respiratory failure in mice. eLife 7:e38455. doi: 10.7554/ELIFE.38455

van der Kooy, D., and Koda, L. (1983). Organization of the projections of a circumventricular organ: the area postrema in the rat. J. Comp. Neurol. 219, 328-338. doi: 10.1002/CNE.902190307

Veinante, P., Jacquin, M. F., Desche^nes, M., and Desche^nes, D. (2000). Thalamic projections from the whisker-sensitive regions of the spinal trigeminal complex in the rat. J. Comp. Neurol 420, 233-243.

Vogel-Höpker, A., and Rohrer, H. (2002). The specification of noradrenergic locus coeruleus (LC) neurones depends on bone morphogenetic proteins (BMPs). Development 129, 983-991. doi: 10.1242/DEV.129.4.983

Walsh, F., and Doherty, P. (1997). Neural cell adhesion molecules of the immunoglobulin superfamily: role in axon growth and guidance. Annu. Rev. Cell Dev. Biol. 13, 425-456. doi: 10.1146/ANNUREV.CELLBIO.13. 1.425

Wang, V. Y., Rose, M. F., and Zoghbi, H. Y. (2005). Math1 expression redefines the rhombic lip derivatives and reveals novel lineages within the brainstem and cerebellum. Neuron 48, 31-43. doi: 10.1016/J.NEURON.2005.08.024

Wang, X., Kohl, A., Yu, X., Zorio, D., Klar, A., Sela-Donenfeld, D., et al. (2020). Temporal-specific roles of fragile X mental retardation protein in the development of the hindbrain auditory circuit. Development 147:dev188797. doi: 10.1242/DEV.188797

Watson, C., Bartholomaeus, C., and Puelles, L. (2019). Time for Radical Changes in Brain Stem Nomenclature-Applying the Lessons From Developmental Gene Patterns. Front. Neuroanat. 13:10. doi: 10.3389/fnana.2019.00010

Watson, C., Shimogori, T., and Puelles, L. (2017). Mouse Fgf8-Cre-LacZ lineage analysis defines the territory of the postnatal mammalian isthmus. J. Comp. Neurol. 525, 2782-2799. doi: 10.1002/cne.24242

Weese-Mayer, D., Berry-Kravis, E., and Marazita, M. (2005). In pursuit (and discovery) of a genetic basis for congenital central hypoventilation syndrome. Respir. Physiol. Neurobiol. 149, 73-82. doi: 10.1016/J.RESP.2005. 06.010

Weisinger, K., Kayam, G., Missulawin-Drillman, T., and Sela-Donenfeld, D. (2010). Analysis of expression and function of FGF-MAPK signaling components in the hindbrain reveals a central role for FGF3 in the regulation of Krox20, mediated by Pea3. Dev. Biol. 344, 881-895. doi: 10.1016/j.ydbio.2010.06.001
Weisinger, K., Wilkinson, D. G., and Sela-Donenfeld, D. (2008). Inhibition of BMPs by follistatin is required for FGF3 expression and segmental patterning of the hindbrain. Dev. Biol. 324, 213-225. doi: 10.1016/j.ydbio.2008.09.005

Williams, J., Murphy, D., Reynolds, K., Welch, S., and King, M. (1996). Demonstration of a bilateral projection from the rostral nucleus of the solitary tract to the medial parabrachial nucleus in rat. Brain Res. 737, 231-237.

Wilson, S., Shafer, B., Lee, K., and Dodd, J. (2008). A molecular program for contralateral trajectory: Rig-1 control by LIM homeodomain transcription factors. Neuron 59, 413-424. doi: 10.1016/J.NEURON.2008.07.020

Wingate, R., and Hatten, M. E. (1999). The role of the rhombic lip in avian cerebellum development. Development 126, 4395-4404.

Wolman, M. A., Regnery, A. M., Becker, T., Becker, C. G., and Halloran, M. C. (2007). Semaphorin3D regulates axon-axon interactions by modulating levels of L1 Cell Adhesion Molecule. J. Neurosci. 27, 9653-9663. doi: 10.1523/ JNEUROSCI.1741-07.2007

Yamada, M., Terao, M., Terashima, T., Fujiyama, T., Kawaguchi, Y., Nabeshima, Y., et al. (2007). Origin of climbing fiber neurons and their developmental dependence on Ptfla. J. Neurosci. 27, 10924-10934. doi: 10.1523/JNEUROSCI. 1423-07.2007

Yang, J. L. J., Bertolesi, G. E., Hehr, C. L., and McFarlane, S. (2020). Lhx2/9 and Etv1 Transcription Factors have Complementary roles in Regulating the Expression of Guidance Genes slit1 and sema3a. Neuroscience 434, 66-82. doi: 10.1016/J. NEUROSCIENCE.2020.03.021

Yee, K. T., Simon, H. H., Tessier-Lavigne, M., and O'Leary, D. D. M. (1999). Extension of long leading processes and neuronal migration in the mammalian brain directed by the chemoattractant Netrin-1. Neuron 24, 607-622.

Yung, A. R., Druckenbrod, N. R., Cloutier, J. F., Wu, Z., Tessier-Lavigne, M., and Goodrich, L. V. (2018). Netrin-1 Confines Rhombic Lip-Derived Neurons to the CNS. Cell Rep. 22, 1666-1680. doi: 10.1016/J.CELREP.2018.01.068

Zeng, C., Shroff, H., and Shore, S. E. (2011). Cuneate and spinal trigeminal nucleus projections to the cochlear nucleus are differentially associated with vesicular glutamate transporter-2. Neuroscience 176, 142-151. doi: 10.1016/J. NEUROSCIENCE.2010.12.010

Zhou, J., and Shore, S. (2006). Convergence of spinal trigeminal and cochlear nucleus projections in the inferior colliculus of the guinea pig. J. Comp. Neurol. 495, 100-112. doi: 10.1002/CNE.20863

Zhu, Y., Guthrie, S., and Murakami, F. (2006). Ephrin A/EphA controls the rostral turning polarity of a lateral commissural tract in chick hindbrain. Development 133, 3837-3846. doi: 10.1242/DEV.02564

Zijing, L., Hong, L., Xuemei, H., Ling, Y., Hongbin, L., Ruifa, H., et al. (2008). Control of precerebellar neuron development by Olig3 bHLH transcription factor. J. Neurosci. 28, 10124-10133. doi: 10.1523/JNEUROSCI.3769-08.2008

Conflict of Interest: The authors declare that the research was conducted in the absence of any commercial or financial relationships that could be construed as a potential conflict of interest.

Publisher's Note: All claims expressed in this article are solely those of the authors and do not necessarily represent those of their affiliated organizations, or those of the publisher, the editors and the reviewers. Any product that may be evaluated in this article, or claim that may be made by its manufacturer, is not guaranteed or endorsed by the publisher.

Copyright (c) 2021 Hirsch, Kohl, Wang and Sela-Donenfeld. This is an open-access article distributed under the terms of the Creative Commons Attribution License (CC BY). The use, distribution or reproduction in other forums is permitted, provided the original author(s) and the copyright owner(s) are credited and that the original publication in this journal is cited, in accordance with accepted academic practice. No use, distribution or reproduction is permitted which does not comply with these terms. 